\title{
Torsion in graph homology
}

\author{
by \\ Laure Helme-Guizon, Józef H. Przytycki \\ and Yongwu Rong (Washington, DC)
}

\begin{abstract}
Khovanov homology for knots has generated a flurry of activity in the topology community. This paper studies the Khovanov type cohomology for graphs with a special attention to torsion. When the underlying algebra is $\mathbb{Z}[x] /\left(x^{2}\right)$, we determine precisely those graphs whose cohomology contains torsion. For a large class of algebras, we show that torsion often occurs. Our investigation of torsion led to other related general results. Our computation could potentially be used to predict the Khovanov-Rozansky $s l(m)$ homology of knots (in particular $(2, n)$ torus knot). We also predict that our work is connected with Hochschild and Connes cyclic homology of algebras.
\end{abstract}

\section{Contents}

1. Introduction 140

2. The graph cohomology, a quick review 140

3. General results 143

3.1. The Vanishing Theorem 143

3.2. Thickness of the cohomology 145

$\begin{array}{lr}\text { 3.3. The particular case of polygons } & 148\end{array}$

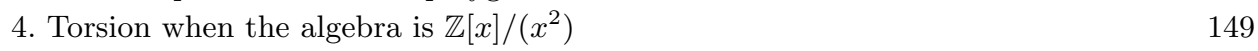

4.1. Facts and observations 149

4.2. The result 151

4.3. The odd cycle case $\quad 152$

4.4. The even cycle case $\quad 155$

4.5. Connection with Khovanov cohomology for links 159

5. Computations for the algebras $\mathcal{A}_{m}$ and their deformations $\quad 161$

5.1. $H_{\mathcal{A}_{m}}^{i, j}$ for a triangle $\quad 162$

$\begin{array}{ll}\text { 5.2. More calculations } & 167\end{array}$

$\begin{array}{ll}\text { 5.3. Deformation of the algebra } \mathcal{A}_{m} & 171\end{array}$

$\begin{array}{ll}\text { References } & 176\end{array}$

2000 Mathematics Subject Classification: Primary 57M27; Secondary 05C15, 55N35.

Key words and phrases: graph, knot, Khovanov homology, graph homology, Hochschild homology, torsion.

Research partially supported by NSF grant DMS-0513918. 
1. Introduction. In [K00], M. Khovanov introduced a graded cohomology theory for knots whose Euler characteristic is the Jones polynomial of the knot. Since then, there has been a great deal of interest in his theory. In [HR04], a cohomology theory for graphs was constructed using the algebra $\mathbb{Z}[x] /\left(x^{2}\right)$. In [HR05], this was generalized by allowing more general algebras. In both papers, the graded Euler characteristic of the homology groups yields the chromatic polynomial, through an appropriate change of variable.

The Euler characteristic is defined in terms of the free part of the cohomology groups. On the other hand, the torsion of these homology groups often demonstrates more interesting properties. In [S04], A. Shumakovitch did an extensive computation and proved, among other things, that nontrivial alternating knots always have $\mathbb{Z}_{2}$-torsion. In [AP04], M. Asaeda and J. Przytycki generalized this to adequate links, and located specific torsion for a large family of links.

Here, we analyze torsion in the graph cohomology introduced in [HR04] and [HR05]. For the algebra $\mathbb{Z}[x] /\left(x^{2}\right)$, we determine precisely those graphs whose cohomology contains torsion in Theorem 21. We also study to what extent this can be extended to more general algebras.

In Section 2, we review the constructions in [HR04] and [HR05]. In Section 3, we prove some thickness type theorem for the cohomology groups. Section 4 contains the Torsion Theorem (Theorem 21) for the algebra $\mathbb{Z}[x] /\left(x^{2}\right)$. In Section 5 , we consider the cohomology groups for more general algebras. Special attention is paid to the algebra $\mathcal{A}_{m}=\mathbb{Z}[x] /\left(x^{m}\right)$ and the graph $P_{v}$, the polygon with $v$ vertices. Subsection 5.3 contains a calculation of cohomology for $\mathcal{A}=\mathbb{Z}[x] / \mathbb{I}$, where $\mathbb{I}$ is an ideal, in the case of a triangle. We end Section 5 with some general comments.

2. The graph cohomology, a quick review. We briefly review our constructions in [HR04] and [HR05]. Recall that a graded $\mathbb{Z}$-algebra $\mathcal{A}$ is a $\mathbb{Z}$-algebra with direct sum decomposition $\mathcal{A}=\bigoplus_{j=0}^{\infty} A_{j}$ into $\mathbb{Z}$-submodules such that $a_{i} a_{j} \in A_{i+j}$ for all $a_{i} \in A_{i}$ and $a_{j} \in A_{j}$. The elements of $A_{j}$ are called homogeneous of degree $j$.

Since $\mathcal{A}$ can be considered as a $\mathbb{Z}$-module, its graded dimension is defined as follows. Let $M=\bigoplus_{j=0}^{\infty} M_{j}$ be a graded $\mathbb{Z}$-module where $M_{j}$ denotes the set of homogeneous elements of degree $j$ of $M$. Recall that the free rank of a $\mathbb{Z}$-module $M$ is $\operatorname{dim}_{\mathbb{Q}}\left(M \otimes_{\mathbb{Z}} \mathbb{Q}\right)$ and is denoted by $\operatorname{rank}(M)$. Assume that $\operatorname{rank}\left(M_{j}\right)<\infty$ for each $j$. The graded dimension of $M$ is the power series

$$
q \operatorname{dim} M:=\sum_{j=0}^{\infty} q^{j} \operatorname{rank}\left(M_{j}\right) .
$$


We will use $\{\cdot\}$ to denote the degree shift in graded $\mathbb{Z}$-modules. For example, $\mathbb{Z}^{3}\{2\}$ denotes the rank three free abelian group whose elements are of degree two.

From now on, we work with algebras that satisfy the following conditions:

Assumptions 1 . $\mathcal{A}=\bigoplus_{i=0}^{\infty} A_{i}$ is a commutative, graded $\mathbb{Z}$-algebra with identity such that each $A_{i}$ is free of finite rank.

Note that these assumptions can sometimes be relaxed. For instance, if there is no identity or if the $A_{i}$ 's are not free, the construction can still be made but some properties will have to be modified.

Now, we fix an algebra $\mathcal{A}$ satisfying Assumptions 1 . Let $G$ be a graph and $E=E(G)$ be the edge set of $G$. Let $n=|E|$ be the cardinality of $E$. We fix an ordering on $E$ and denote the edges by $e_{1}, \ldots, e_{n}$. Consider the $n$ dimensional cube $\{0,1\}^{E}=\{0,1\}^{n}$. Each vertex $\alpha$ of this cube corresponds to a subset $s=s_{\alpha}$ of $E$, where $e_{i} \in s_{\alpha}$ if and only if $\alpha_{i}=1$. The height $|\alpha|$ of $\alpha$ is defined by $|\alpha|=\sum \alpha_{i}$, which is also equal to the number of edges in $s_{\alpha}$.

With each vertex $\alpha$ of the cube, we associate the graded $\mathbb{Z}$-module $C^{\alpha}(G)$ as follows. Consider $[G: s]$, the graph with vertex set $V(G)$ and edge set $s$. We assign a copy of $\mathcal{A}$ to each component of $[G: s]$ and then take the tensor product over the components. Let $C^{\alpha}(G)$ be the resulting graded $\mathbb{Z}$-module, with the grading induced from $\mathcal{A}$. Therefore, $C^{\alpha}(G) \cong \mathcal{A}^{\otimes k}$ where $k$ is the number of components of $[G: s]$. We define the $i$ th chain group of our complex to be $C^{i}(G):=\bigoplus_{|\alpha|=i} C^{\alpha}(G)$.

Next, we describe the differential maps $d^{i}: C^{i}(G) \rightarrow C^{i+1}(G)$. These will be expressed in terms of the per-edge maps. Each edge $\xi$ of $\{0,1\}^{E}$ can be labeled by a sequence in $\{0,1, *\}^{E}$ with exactly one $*$. The tail of the edge, denoted by $\alpha_{1}$, is obtained by setting $*=0$; and the head, denoted by $\alpha_{2}$, is obtained by setting $*=1$. The height $|\xi|$ is defined to be the height of its tail, which is also equal to the number of 1 's in $\xi$. For $j=1$ and 2 , the $\mathbb{Z}$-module $C^{\alpha_{j}}(G)$ is $\mathcal{A}^{\otimes k_{j}}$ where $k_{j}$ is the number of connected components of $\left[G: s_{j}\right]$ (here $s_{j}$ stands for $s_{\alpha_{j}}$ ). Let $e$ be the edge with $s_{2}=s_{1} \cup\{e\}$. The per-edge map $d_{\xi}: C^{\alpha_{1}}(G) \rightarrow C^{\alpha_{2}}(G)$ is defined as follows.

If $e$ joins a component of $\left[G: s_{1}\right]$ to itself, then $k_{1}=k_{2}$ and the components of $\left[G: s_{1}\right]$ and the components of $\left[G: s_{2}\right]$ naturally correspond to each other. We then let $d_{\xi}$ be the identity map.

If $e$ joins two different components of $\left[G: s_{1}\right]$, say $E_{1}$ to $E_{2}$ where $E_{1}, E_{2}, \ldots, E_{k_{1}}$ are the components of $\left[G: s_{1}\right]$, then $k_{2}=k_{1}-1$ and the components of $\left[G: s_{2}\right]$ are $E_{1} \cup E_{2} \cup\{e\}, E_{3}, \ldots, E_{k_{1}}$. We define $d_{\xi}$ to be the identity map on the tensor factors coming from $E_{3}, \ldots, E_{k_{1}}$, and the multiplication map $\mathcal{A} \otimes \mathcal{A} \rightarrow \mathcal{A}$ sending $x \otimes y$ to $x y$ on the remaining tensor factors. 
The differential $d^{i}: C^{i}(G) \rightarrow C^{i+1}(G)$ is then defined by

$$
d^{i}=\sum_{|\xi|=i}(-1)^{\xi} d_{\xi}
$$

where $(-1)^{\xi}=-1$ (resp. 1) if the number of 1's in $\xi$ before $*$ is odd (resp. even).

It was proved in [HR05] that this defines a graded cochain complex $\mathcal{C}$ whose graded cohomology groups $H^{i}$ are invariants of the graph (i.e. independent of the ordering of the edges), and whose graded Euler characteristic is the chromatic polynomial of $G$ evaluated at $\lambda=q \operatorname{dim} \mathcal{A}$, i.e.

$$
\begin{aligned}
\chi_{q}(\mathcal{C}) & =\sum_{0 \leq i \leq n}(-1)^{i} q \operatorname{dim}\left(H^{i}\right)=\sum_{0 \leq i \leq n}(-1)^{i} q \operatorname{dim}\left(C^{i}\right) \\
& =P_{G}(q \operatorname{dim} \mathcal{A}) .
\end{aligned}
$$

The cochain complex $\mathcal{C}$ splits into a sequence of cochain complexes, one for each degree $j$. More precisely, let $\mathcal{C}=0 \rightarrow C^{0} \rightarrow C^{1} \rightarrow \cdots \rightarrow C^{n} \rightarrow 0$ be a graded cochain complex with a degree preserving differential. Decomposing elements of each cochain group by degree yields $C^{i}=\bigoplus_{j \geq 0} C^{i, j}$, where $C^{i, j}$ is the set of homogeneous elements of $C^{i}$ of degree $j$. Since the differential is degree preserving, the restriction to elements of degree $j$, i.e. $0 \rightarrow C^{0, j} \rightarrow$ $C^{1, j} \rightarrow \cdots \rightarrow C^{n, j} \rightarrow 0$, is a cochain complex denoted by $\mathcal{C}^{j}$. It is clear that $\mathcal{C}$ is the direct sum of these cochain complexes.

It is also convenient to use the notion of "enhanced states". When the algebra is $\mathbb{Z}[x] /\left(x^{2}\right)$, an enhanced state of a graph $G$ is $S=(s, c)$, where $s \subseteq$ $E(G)$ and $c$ is an assignment of colors 1 or $x$ to each connected component of the spanning subgraph $[G: s]$. Such enhanced states form a basis for the chain groups. Furthermore, the differential map $d$ can be described explicitly in terms of enhanced states. Details can be found in [HR04]. This can be generalized to more general algebras with a given generating set for its additive group, with proper translations for any possible relations on the generators.

An interesting property of these cohomology groups is a long exact sequence described below, which corresponds to the deletion-contraction rule for the chromatic polynomial. Let $e$ be an edge of the graph $G$. Recall that $G-e$ denotes the graph obtained by deleting $e$ from $G$, and $G / e$ denotes the graph obtained by contracting $e$ to a point. We have

\section{THEOREM 2.}

(a) For each $i, j$, there is a short exact sequence of graded chain homomorphisms: $0 \rightarrow C^{i-1, j}(G / e) \stackrel{\alpha}{\rightarrow} C^{i, j}(G) \stackrel{\beta}{\rightarrow} C^{i, j}(G-e) \rightarrow 0$, and therefore, 
(b) it induces a long exact sequence of cohomology groups: $0 \rightarrow H^{0, j}(G)$ $\rightarrow H^{0, j}(G-e) \rightarrow H^{0, j}(G / e) \rightarrow H^{1, j}(G) \rightarrow \cdots \rightarrow H^{i, j}(G) \stackrel{\beta^{*}}{\rightarrow}$
$H^{i, j}(G-e) \rightarrow H^{i, j}(G / e) \rightarrow \cdots$.

This long exact sequence has proved to be a useful computational tool, as we will see in the next several sections.

Another useful result from [HR05] is about pendant edges. Recall that a pendant vertex in a graph is a vertex of degree one, and a pendant edge is an edge connecting a pendant vertex to another vertex.

Proposition 3. Let $\mathcal{A}$ be an algebra satisfying Assumptions 1 . It can be written $\mathcal{A}=\mathbb{Z} 1_{\mathcal{A}} \oplus \mathcal{A}^{\prime}$ as a $\mathbb{Z}$-module, where $\mathbb{Z} 1_{\mathcal{A}}$ is generated by the identity of $\mathcal{A}$ and $\mathcal{A}^{\prime}$ is a submodule of $\mathcal{A}$. If $e$ is a pendant edge of $G$, then $H^{i}(G) \cong H^{i}(G / e) \otimes \mathcal{A}^{\prime}$.

3. General results. Theorems 4,10,12 and Corollary 13 below indicate which cohomology groups of a graph may be non-trivial. They generalize similar results that can be found in [H05] in the case $\mathcal{A}=\mathcal{A}_{2}$.

\subsection{The Vanishing Theorem}

TheOREM 4. Let $G=G_{1} \sqcup G_{2}$, where $G_{2}$ is the union of the components of $G$ that are trees, and $G_{1}$ is the union of the remaining components. Let $v_{i}$ be the number of vertices of $G_{i}$ and $\mu_{i}$ be the number of components of $G_{i}(i=1,2)$. For any algebra $\mathcal{A}$ satisfying Assumptions 1 , we have

(a) $H_{\mathcal{A}}^{i}(G) \neq 0 \Rightarrow 0 \leq i \leq v_{1}-2 \mu_{1}$,

(b) $\operatorname{Tor}\left(H_{\mathcal{A}}^{i}(G)\right) \neq 0 \Rightarrow 1 \leq i \leq v_{1}-2 \mu_{1}$.

In particular, if a graph has no isolated vertices, we get the following more convenient inequality.

Corollary 5. Let $G$ be a $\mu$-component graph with $v$ vertices. If $G$ has no isolated vertices, then $H_{\mathcal{A}}^{i}(G) \neq 0 \Rightarrow 0 \leq i \leq v-2 \mu$.

Proof. We use the notations of Theorem 4. We have $v-2 \mu=v_{1}-2 \mu_{1}+$ $v_{2}-2 \mu_{2}$. By assumption, each component of $G_{2}$ has at least two vertices, therefore $v_{2}-2 \mu_{2} \geq 0$ and this implies $v-2 \mu \geq v_{1}-2 \mu_{1}$.

REMark 6. Theorem 4 implies the following result of [H05]: If $G$ is a $\mu$ component graph with $v$ vertices, then $H_{\mathcal{A}}^{i}(G) \neq 0$ implies $0 \leq i \leq v-\mu-1$. In particular, if $v \geq 2$, then $H_{\mathcal{A}}^{i}(G) \neq 0$ implies $0 \leq i \leq v-2$. This observation was first made by Michael and Sergei Chmutov [C05] in the case when the graph is connected and $\mathcal{A}=\mathcal{A}_{2}$.

REMARK 7. The inequality in Theorem 4 is sharp for graphs that are forests, polygons, or their disjoint unions. But if a (non-forest) graph contains a pendant edge, we can improve the previous result. For example, we 
have

Corollary 8 (of Theorem 4).

(i) $H^{v_{G}-k-1}(G)=0$ if $G$ has $k$ pendant edges and $v_{G}-k-1 \geq 1$.

(ii) If $G$ has a pendant edge in a non-tree component, then $H_{\mathcal{A}}^{i}(G) \neq 0$ $\Rightarrow 0 \leq i \leq v_{1}-2 \mu_{1}-1$.

Proof. (i) Remark 6 implies that for any graph $G$ with $v_{G} \geq 2, H_{\mathcal{A}}^{i}(G)$ $\neq 0$ implies $0 \leq i \leq v_{G}-2$. Using Proposition 3, we see that if a graph $Y^{p}$ is obtained from $Y$ by adding a pendant edge then $H_{\mathcal{A}}^{i, *}\left(Y^{p}\right)=0$ whenever $H_{\mathcal{A}}^{i, *}(Y)=0$. Combining these two results, we see that $H_{\mathcal{A}}^{v-2, *}\left(Y^{p}\right)=0$, where $v$ is the number of vertices of $Y^{p}$. We get the announced result by induction on the number of pendant edges.

(ii) Let $e$ be a given pendant edge in a non-tree component. By Proposition 3, we have $H_{\mathcal{A}}^{i}(G) \cong H_{\mathcal{A}}^{i}(G / e) \otimes \mathcal{A}^{\prime}$. Therefore, $H_{\mathcal{A}}^{i}(G) \neq 0$ implies $H_{\mathcal{A}}^{i}(G / e) \neq 0$, which implies $i \leq v_{1}(G / e)-2 \mu_{1}(G / e)=v_{1}(G)-2 \mu_{1}(G)-1$.

Proof of Theorem 4. (a) We will prove the inequality by induction on $n$, where $n$ is the number of edges in $G$.

If $n=0, G$ is the null graph with $v_{2}$ vertices. We have $v_{1}=\mu_{1}=0$. It is well known that $H_{\mathcal{A}}^{0}(G) \cong \mathcal{A}^{\otimes v_{2}}$ and $H_{\mathcal{A}}^{i}(G)=0$ for all $i>0$ [HR05]. Thus the inequality holds.

Suppose the inequality holds for all graphs with no more than $n$ edges. Now let $G$ be a graph with $n$ edges.

If $G$ is a forest, then it is well known that $H^{i}(G)=0$ for all $i>0$ [HR05]. Thus the inequality $0 \leq i \leq v_{1}-2 \mu_{1}$ becomes $0 \leq 0 \leq 0$.

If $G$ has multiple edges, deleting one such edge $e$ yields a graph $G-e$ with one edge less. Note that $v_{1}(G-e)=v_{1}(G)$ and $\mu_{1}(G-e)=\mu_{1}(G)$. Since cohomology groups remain the same when we delete a multiple edge, applying induction $G-e$ proves that the inequality holds for $G$.

If $G$ has a loop, the inequality holds trivially since $H_{\mathcal{A}}^{i}(G)=0$ for all $i$.

Now, we assume that $G$ is not a forest, and has no loops or multiple edges. It follows that there is a cycle of length 3 or greater. Let $e$ be an edge in this cycle. Assume that $i>v_{1}-2 \mu_{1}$. Applying the exact sequence on $(G, e)$, we have

$$
\cdots \rightarrow H_{\mathcal{A}}^{i-1}(G / e) \rightarrow H_{\mathcal{A}}^{i}(G) \rightarrow H_{\mathcal{A}}^{i}(G-e) \rightarrow \cdots .
$$

For the graph $G / e$, we have $v_{1}(G / e)=v_{1}(G)-1, \mu_{1}(G / e)=\mu_{1}(G)$, thus $v_{1}(G / e)-2 \mu_{1}(G / e)=v_{1}(G)-2 \mu_{1}(G)-1<i-1$, which implies $H_{\mathcal{A}}^{i-1}(G / e)=0$ by induction.

For the graph $G-e$, we have $v_{1}(G-e)=v_{1}(G)$ and $\mu_{1}(G-e)=$ $\mu_{1}(G)$. Thus $v_{1}(G-e)-2 \mu_{1}(G-e)=v_{1}(G)-2 \mu_{1}(G)<i$, which implies $H_{\mathcal{A}}^{i}(G-e)=0$.

Therefore, we have $H_{\mathcal{A}}^{i}(G)=0$ by the exact sequence. 
(b) By (a), the only thing we need to show is that $H^{0}(G)$ contains no torsion. But this follows since $H^{0}(G)=\operatorname{ker}\left(d^{0}\right)$, which is a subgroup of the free abelian group $C^{0}$.

In the next subsection, we show that if we restrict the class of algebras we deal with, we can refine the result of Theorem 4 .

3.2. Thickness of the cohomology. Indeed, this subsection contains the following two results: Theorem 10 shows that under the mild assumption that the algebra is pointed (see Definition 9 below), the cohomology groups corresponding to "small" values $i$ and $j$ are trivial.

Theorem 12 shows that under the assumption that the algebra is of degree less than or equal to $m$, the cohomology groups corresponding to "large" values $i$ and $j$ are trivial.

We then combine these results to determine which cohomology groups may be non-trivial when $\mathcal{A}=\mathcal{A}_{m}$.

Definition 9 . We say that a graded $\mathbb{Z}$-algebra is pointed if it has a direct sum decomposition into $\mathbb{Z}$-submodules of the form $\mathcal{A}=\mathbb{Z} 1 \oplus \mathcal{A}^{\prime}$ where all the homogeneous elements of $\mathcal{A}^{\prime}$ have degree greater than or equal to 1 .

TheOREM 10. Let $G$ be a $\mu$-component graph with $v$ vertices. Let $\mathcal{A}$ be a pointed $\mathbb{Z}$-algebra satisfying Assumptions 1 . We have

(a) $H_{\mathcal{A}}^{i, j}(G) \neq 0 \Rightarrow i+j \geq v-\mu$.

(b) $\operatorname{Tor}\left(H_{\mathcal{A}}^{i, j}(G)\right) \neq 0 \Rightarrow i+j \geq v-\mu+1$.

Proof. Let $n$ be the number of edges of $G$. We argue by induction on $n$, where our induction hypothesis consists of statements (a) and (b).

Initial step. If $n=0, G$ is $N_{v}$, the null graph on $v$ vertices. The fact that the theorem holds for any forest is shown below (without using induction) so we will not repeat the proof here.

Induction step. Assume the induction hypothesis holds for all $n^{\prime}<n$.

If $G$ has a loop, the induction hypothesis is satisfied, so we can assume from now on that $G$ has no loop.

If $G$ has multiple edges, the graph $G^{\prime}$ obtained from $G$ by replacing multiple edges by single edges has fewer edges so we can use the induction hypothesis for $G^{\prime}$. Since $G$ and $G^{\prime}$ have the same cohomology groups, the same number of vertices and the same number of components, this proves the result for $G$. From now on in this proof, we assume that $G$ has no loops and no multiple edges.

CASE 1: $G$ is a forest. Assume $G$ is a forest made of $\mu$ trees with a total of $n$ edges and denote it $F_{\mu, n}$. Note that $F_{\mu, n}$ has $\mu+n$ vertices. The cohomology 
groups of $F_{\mu, n}$ are obtained from the cohomology groups of $N_{\mu}$, the graph with $\mu$ vertex and no edges, by adding $n$ pendant edges. By Theorem 3 , the cohomology groups of $F_{\mu, n}$ are trivial except for $H_{\mathcal{A}}^{0}\left(F_{\mu, n}\right) \cong \mathcal{A}^{\otimes \mu} \otimes\left(\mathcal{A}^{\prime}\right)^{\otimes n}$ where $\mathcal{A}^{\prime}$ satisfies $\mathcal{A}=\mathbb{Z} 1 \oplus \mathcal{A}^{\prime}$ with the degree of all the homogeneous elements of $\mathcal{A}^{\prime}$ greater than or equal to 1 (since $\mathcal{A}$ is pointed). Hence the degrees $j$ of the homogeneous elements of $H_{\mathcal{A}}^{0}\left(F_{\mu, n}\right)$ are greater than or equal to $n$. Substituting $n=v-\mu$ in the above yields $0+j \geq v-\mu$.

Also, all the other cohomology groups are trivial and there is no torsion so the induction hypothesis is satisfied (without using induction).

CASE 2: $G$ is a not a forest. Since we assumed that $G$ has no loop and no multiple edges, $G$ not being a forest means it contains a cycle of order $\geq 3$. Let $e$ be an edge on this cycle. The edge $e$ is not an isthmus so $G-e$ also has $\mu$ components. Of course, $G / e$ has $\mu$ components whether $e$ is an isthmus or not. Therefore, $\mu=\mu_{G}=\mu_{G-e}=\mu_{G / e}$.

The exact sequence on $(G, e)$ yields

$$
\cdots \rightarrow H_{\mathcal{A}}^{i-1, j}(G-e) \rightarrow H_{\mathcal{A}}^{i-1, j}(G / e) \rightarrow H_{\mathcal{A}}^{i, j}(G) \rightarrow H_{\mathcal{A}}^{i, j}(G-e) \rightarrow \cdots
$$

We do not have to worry about having $i-1 \geq 0$ if we define the cohomology groups with negative heights to be the trivial group.

Since $G-e$ and $G / e$ each have one edge less than $G$, the induction hypothesis applies to them.

$\Delta$ Assume that $i+j<v_{G}-\mu_{G}$. We need to show that $H_{\mathcal{A}}^{i, j}(G)=0$. The exact sequence on $(G, e)$ gives

$$
\cdots \rightarrow H_{\mathcal{A}}^{i-1, j}(G / e) \rightarrow H_{\mathcal{A}}^{i, j}(G) \rightarrow H_{\mathcal{A}}^{i, j}(G-e) \rightarrow \cdots .
$$

The inequality $i+j<v_{G}-\mu_{G}=v_{G-e}-\mu_{G-e}$ implies $H_{\mathcal{A}}^{i, j}(G-e)=0$ by part (a) of the induction hypothesis. Also $i+j<v_{G}-\mu_{G}$ implies $(i-1)+j<$ $\left(v_{G}-1\right)-\mu_{G}=v_{G / e}-\mu_{G / e}$ so $H_{\mathcal{A}}^{i-1, j}(G / e)=0$ by (a) again. Therefore the sequence $0 \rightarrow H_{\mathcal{A}}^{i, j}(G) \rightarrow 0$ is exact so $H_{\mathcal{A}}^{i, j}(G)=0$.

$\Delta$ Assume that $i+j<v_{G}+1-\mu_{G}$. We need to show that $\operatorname{Tor}\left(H_{\mathcal{A}}^{i, j}(G)\right)=0$. The exact sequence on $(G, e)$ gives

$$
\cdots \rightarrow H_{\mathcal{A}}^{i-1, j}(G-e) \rightarrow H_{\mathcal{A}}^{i-1, j}(G / e) \rightarrow H_{\mathcal{A}}^{i, j}(G) \rightarrow H_{\mathcal{A}}^{i, j}(G-e) \rightarrow \cdots .
$$

The inequality $i+j<v_{G}+1-\mu_{G}$ implies $(i-1)+j<v_{G}-\mu_{G}=v_{G-e}-\mu_{G-e}$ so $H_{\mathcal{A}}^{i-1, j}(G-e)=0$ by part (a) of the induction hypothesis. The inequality $i+j<v_{G}+1-\mu_{G}=v_{G-e}+1-\mu_{G-e}$ implies that $H_{\mathcal{A}}^{i, j}(G-e)$ is torsion free by part (b) of the induction hypothesis. Also $i+j<v_{G}+1-\mu_{G}$ implies $(i-1)+j<v_{G}-\mu_{G}=v_{G / e}+1-\mu_{G / e}$ so $H_{\mathcal{A}}^{i-1, j}(G / e)$ is torsion free by (b). Therefore the exact sequence is of the form

$$
\cdots \rightarrow 0 \rightarrow F \rightarrow H_{\mathcal{A}}^{i, j}(G) \rightarrow F^{\prime} \rightarrow \cdots
$$


where $F$ and $F^{\prime}$ are torsion free abelian groups. Note that we are working with finitely generated abelian groups so free and torsion free are equivalent notions.

The following claim shows that this is enough to prove that $H_{\mathcal{A}}^{i, j}(G)$ is free.

Claim. If $H$ is an abelian group in an exact sequence of the form

$$
0 \rightarrow F \rightarrow H \stackrel{h}{\rightarrow} F^{\prime} \rightarrow \cdots
$$

where $F$ and $F^{\prime}$ are free abelian groups, then $H$ is free.

Proof of the claim. The above exact sequence induces the short exact sequence

$$
0 \rightarrow F \rightarrow H \stackrel{h}{\rightarrow} \operatorname{Im}(h) \rightarrow 0
$$

that splits because $\operatorname{Im}(h)$ is free. Therefore, $H \cong F \oplus \operatorname{Im}(h)$, which proves that $H$ is free.

We now replace the hypothesis that the algebra is pointed by the hypothesis that it is of degree less than or equal to $m$.

Definition 11. We say that a graded $\mathbb{Z}$-algebra is of degree less than or equal to $m$ if all the homogeneous elements of $\mathcal{A}$ have degree less than or equal to $m$.

THEOREM 12. Let $G$ be a graph, not necessarily connected, with $v$ vertices. Let $\mathcal{A}$ be a $\mathbb{Z}$-algebra of degree less than or equal to $m-1$ satisfying Assumptions 1. We have $H_{\mathcal{A}}^{i, j}(G) \neq 0 \Rightarrow(m-1) i+j \leq(m-1) v$.

Proof. Let $n$ be the number of edges of $G$. We are going to prove the result by induction on $n$.

Initial step. If $n=0, G$ is $N_{v}$, the null graph on $v$ vertices. In particular, $G$ is a forest. The fact that the theorem holds for any forest is shown below (without using induction) so we won't repeat the proof here.

Induction step. Let $n \geq 1$. Assume the induction hypothesis holds for $n-1$. Assume that $(m-1) i+j>(m-1) v_{G}$. We need to show that $H_{\mathcal{A}}^{i, j}(G)=0$. Let $e$ be an edge of $G$. The exact sequence on $(G, e)$ gives

$$
\cdots \rightarrow H_{\mathcal{A}}^{i-1, j}(G / e) \rightarrow H_{\mathcal{A}}^{i, j}(G) \rightarrow H_{\mathcal{A}}^{i, j}(G-e) \rightarrow \cdots .
$$

Note that since $G-e$ and $G / e$ have one edge less than $G$, the induction hypothesis applies to them. The inequality $(m-1) i+j>(m-1) v_{G}=$ $(m-1) v_{G-e}$ implies $H_{\mathcal{A}}^{i, j}(G-e)=0$ by the induction hypothesis. Also $(m-1) i+j>(m-1) v_{G}$ implies $(m-1)(i-1)+j>(m-1)\left(v_{G}-1\right)=$ $(m-1) v_{G / e}$ so $H^{i-1, j}(G / e)=0$ by the induction hypothesis. The sequence $0 \rightarrow H_{\mathcal{A}}^{i, j}(G) \rightarrow 0$ is exact so $H_{\mathcal{A}}^{i, j}(G)=0$. 
We define the $\mathbb{Z}$-algebra $\mathcal{A}_{m}$ by $\mathcal{A}_{m}:=\mathbb{Z}[x] /\left(x^{m}\right)$. For this particular algebra we can combine the previous theorems to get

Corollary 13. Let $G$ be a $\mu$-component graph with $v$ vertices. If $G$ has no isolated vertices, then:

1. $H_{\mathcal{A}_{m}}^{i, j}(G) \neq 0 \Rightarrow\left\{\begin{array}{l}0 \leq i \leq v-2 \mu \\ i+j \geq v-\mu \\ (m-1) i+j \leq(m-1) v\end{array}\right.$

2. $\operatorname{Tor}\left(H_{\mathcal{A}_{m}}^{i, j}(G)\right) \neq 0 \Rightarrow\left\{\begin{array}{l}1 \leq i \leq v-2 \mu \\ i+j \geq v+1-\mu \\ (m-1) i+j \leq(m-1) v\end{array}\right.$

This shows the thickness of the region where $H^{i, j}(G)$ is supported. An illustration is given in Figure 1. In the case when $m=2$, and $G$ is connected, this was also observed by M. Chmutov and S. Chmutov [C05].

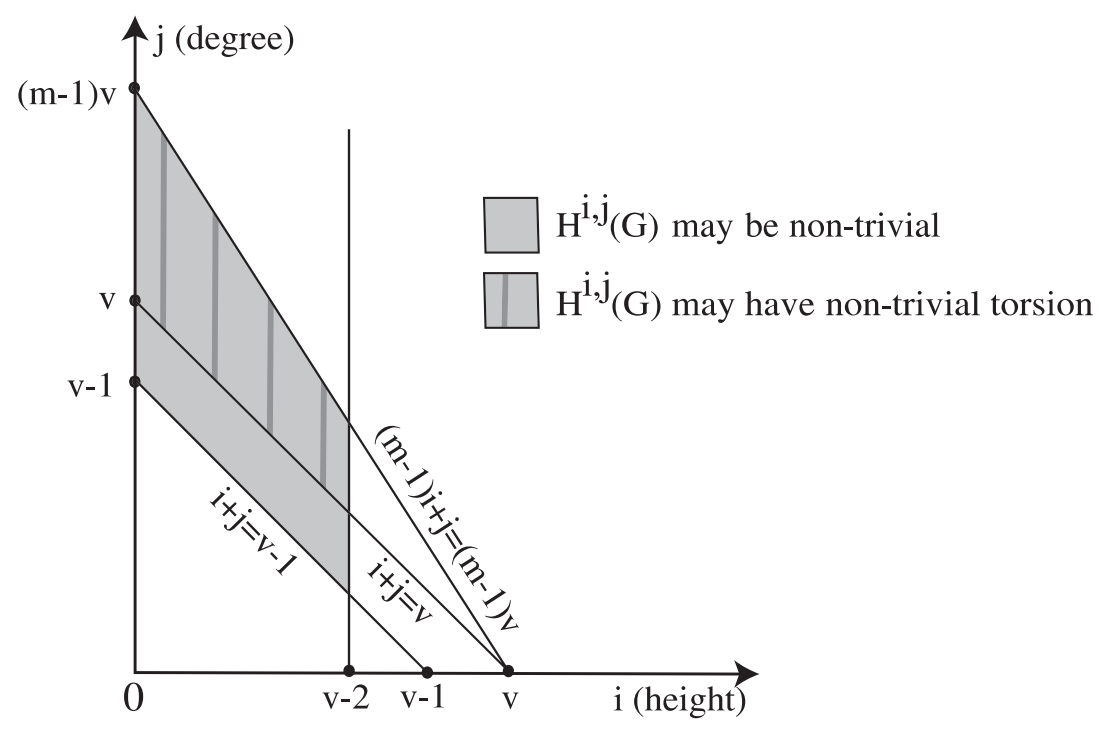

Fig. 1. Thickness restrictions when $\mathcal{A}=\mathcal{A}_{m}$ for a connected graph

3.3. The particular case of polygons. For $n \geq 3$, we denote by $P_{n}$ the polygonal graph with $n$ edges (and $n$ vertices). We can extend this definition in a natural way to the "degenerate" cases $n=1$ and $n=2$ the following way. Let $P_{1}$ be the graph with one vertex and a loop attached to it, and let $P_{2}$ be the graph with two vertices connected by two edges. 
Lemma 14. Let $P_{n}$ be the polygon with $n$ edges and let $\mathcal{A}$ be an algebra satisfying Assumptions 1. Then

$$
H_{\mathcal{A}}^{i+1}\left(P_{n+1}\right)=H_{\mathcal{A}}^{i}\left(P_{n}\right) \quad \text { if } i \geq 1 \text { and } n \geq 1 .
$$

Proof. In [HR05], it is shown that an algebra $\mathcal{A}$ satisfying Assumptions 1 can be written $\mathcal{A}=\mathbb{Z} 1 \oplus \mathcal{A}^{\prime}$. With these notations, the cohomology groups for a tree $T_{n}$ with $n$ edges are known to be (see [HR05])

$$
H_{\mathcal{A}}^{0}\left(T_{n}\right)=\mathcal{A} \otimes \mathcal{A}^{\prime \otimes n}, \quad H_{\mathcal{A}}^{i}\left(T_{n}\right)=0 \quad \text { if } i \neq 0 .
$$

Fix $n \geq 1$ and $i \geq 1$, and let $e$ be an edge of $P_{n+1}$. Applying the above result for trees to the exact sequence on $\left(P_{n+1}, e\right)$ yields

$$
\begin{aligned}
& \begin{array}{cccc}
G-e & G / e & G & G-e
\end{array} \\
& \cdots \rightarrow H^{i}\left(T_{n}\right) \rightarrow H^{i}\left(P_{n}\right) \rightarrow H^{i+1}\left(P_{n+1}\right) \rightarrow H^{i+1}\left(T_{n}\right) \rightarrow \cdots \\
& \|\quad\| \\
& 0 \\
& 0
\end{aligned}
$$

This proves the expected result.

By induction, we get

Corollary 15. Let $P_{n}$ be the polygon with $n$ edges and let $\mathcal{A}$ be an algebra satisfying Assumptions 1. Then

$$
H_{\mathcal{A}}^{i}\left(P_{n}\right)= \begin{cases}H_{\mathcal{A}}^{1}\left(P_{n-i+1}\right) & \text { if } i \leq n, \\ 0 & \text { otherwise. }\end{cases}
$$

This shows that determining the cohomology groups at height 1 is enough to recover all of them. Indeed, the only cohomology groups not determined by the above formula are the ones at height 0 . They can be obtained from the previous ones via the chromatic polynomial since $\sum_{0 \leq i \leq n}(-1)^{i} q \operatorname{dim}\left(H^{i}\right)=$ $P_{G}(q \operatorname{dim} \mathcal{A})$.

4. Torsion when the algebra is $\mathbb{Z}[x] /\left(x^{2}\right)$. In this section, we will focus on the algebra $\mathcal{A}_{2}=\mathbb{Z}[x] /\left(x^{2}\right)$. Thus all cohomology groups will be based on $\mathcal{A}_{2}$. In 4.1 , we review some useful facts. Our main result is stated in 4.2 , and proved in 4.3 and 4.4 .

4.1. Facts and observations. Corollary 13 specialized to $m=2$ gives us some information about the thickness of the cohomology of a connected graph:

COROLlary 16. The cohomology of a connected graph is concentrated along two diagonals, namely $i+j=v$ and $i+j=v-1$. Torsion can only occur on the former. 
The "diagonals" language refers to the way of keeping track of the cohomology groups illustrated in Figure 2. In the array that keeps track of the cohomology groups, the numbers without brackets indicate the number of copies of $\mathbb{Z}$ while a number with brackets of the form $\left[k_{l}\right]$ indicates $k$ copies of $\mathbb{Z}_{l}$. For instance, in the case of the cyclic graph $P_{6}$ (see Figure 2 below), the $\left[1_{2}\right]$ in position $i=2, j=4$ means $H^{2,4}\left(P_{6}\right) \cong \mathbb{Z}_{2}\{4\}$, and the 1 in position $i=2, j=3$ means $H^{2,3}\left(P_{6}\right) \cong \mathbb{Z}\{3\}$, so

$$
H^{2}\left(P_{6}\right) \cong \mathbb{Z}\{3\} \oplus \mathbb{Z}_{2}\{4\} .
$$

This example also illustrates that all the non-trivial cohomology groups have height $i$ such that $0 \leq i \leq 6-2$.
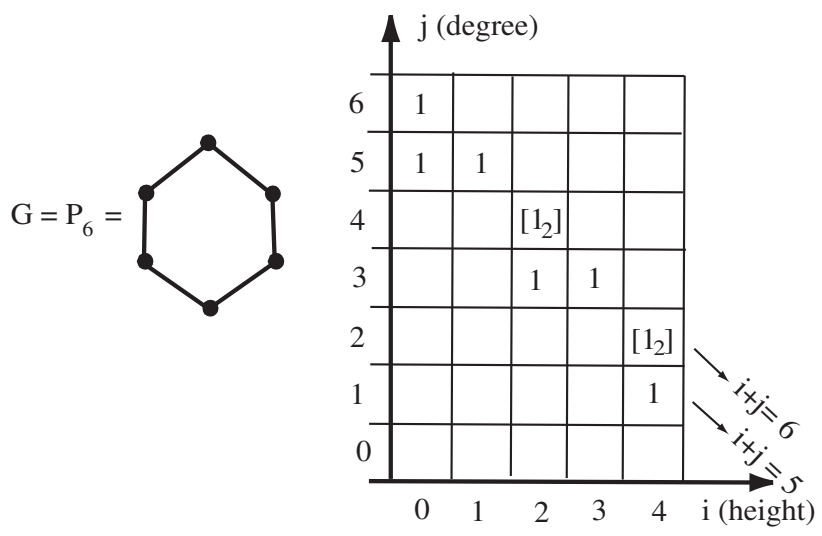

Fig. 2. $H_{\mathcal{A}_{2}}^{*, *}\left(P_{6}\right)$

We now recall some of the facts that were proved in [HR04] and that are going to be useful to determine for which graphs torsion occurs.

FACT 17. The cohomology groups of a forest, so in particular the ones of a tree, are all supported on height zero and do not have torsion.

FACT 18. If the graph has a loop then all the cohomology groups are trivial.

FACT 19. The cohomology groups are unchanged if the multiple edges of a graph are replaced by single edges.

The table below from [HR04] illustrates our computational results (up to $n=6$ and $i=4$ ) for circuit graphs, where $n$ is the length of the cycle and $i$ is the height of the homology group. We denote by $P_{n}$ the circuit graph on $n$ edges where $P$ stands for polygon because the notation $C_{n}$ has already been used for the chain groups. 


\begin{tabular}{l|lllll}
\hline & $H^{0}$ & $H^{1}$ & $H^{2}$ & $H^{3}$ & $H^{4}$ \\
\hline$P_{1}$ & 0 & 0 & 0 & 0 & 0 \\
$P_{2}$ & $\mathbb{Z}\{2\} \oplus \mathbb{Z}\{1\}$ & 0 & 0 & 0 & 0 \\
$P_{3}$ & $\mathbb{Z}\{3\}$ & $\mathbb{Z}_{2}\{2\} \oplus \mathbb{Z}\{1\}$ & 0 & 0 & 0 \\
$P_{4}$ & $\mathbb{Z}\{4\} \oplus \mathbb{Z}\{3\}$ & $\mathbb{Z}\{3\}$ & $\mathbb{Z}_{2}\{2\} \oplus \mathbb{Z}\{1\}$ & 0 & 0 \\
$P_{5}$ & $\mathbb{Z}\{5\}$ & $\mathbb{Z}_{2}\{4\} \oplus \mathbb{Z}\{3\}$ & $\mathbb{Z}\{3\}$ & $\mathbb{Z}_{2}\{2\} \oplus \mathbb{Z}\{1\}$ & 0 \\
$P_{6}$ & $\mathbb{Z}\{6\} \oplus \mathbb{Z}\{5\}$ & $\mathbb{Z}\{5\}$ & $\mathbb{Z}_{2}\{4\} \oplus \mathbb{Z}\{3\}$ & $\mathbb{Z}\{3\}$ & $\mathbb{Z}_{2}\{2\} \oplus \mathbb{Z}\{1\}$ \\
\hline
\end{tabular}

Note that this table illustrates Lemma 14 and Corollary 16. It also illustrates the following general result that appeared in [HR04] and that we repeat here for convenience.

Theorem 20. For $i>0$,

$$
H_{\mathcal{A}_{2}}^{i}\left(P_{n}\right) \cong \begin{cases}\mathbb{Z}_{2}\{n-i\} \oplus \mathbb{Z}\{n-i-1\} & \text { if } n-i \geq 2 \text { and is even }, \\ \mathbb{Z}\{n-i\} & \text { if } n-i \geq 2 \text { and is odd }, \\ 0 & \text { if } n-i \leq 1 .\end{cases}
$$

For $i=0$,

$$
H_{\mathcal{A}_{2}}^{0}\left(P_{n}\right) \cong \begin{cases}\mathbb{Z}\{n\} \oplus \mathbb{Z}\{n-1\} & \text { if } n \text { is even and } n \geq 2, \\ \mathbb{Z}\{n\} & \text { if } n \text { is odd and } n \geq 2, \\ 0 & \text { if } n=1 .\end{cases}
$$

We first note that, for all $n \geq 3, H^{*}\left(P_{n}\right)$ contains torsion.

A closer look at these examples reveals that, in the case of an odd cycle, there seems to always be torsion in $H^{1}\left(P_{v}\right)$ in degree $v-1$, and in the case of an even cycle, there seems to always be torsion in $H^{2}\left(P_{v}\right)$ in degree $v-2$ where $v$ is the number of vertices of the graph. These remarks will guide us for the formulation of the lemmas.

\subsection{The result}

TheOREm 21 (Torsion Theorem). The cohomology $H_{\mathcal{A}_{2}}^{*}(G)$ of a graph $G$ contains a torsion part if and only if $G$ has no loops and contains a cycle of order greater than or equal to 3 . In this case, if the cycle has odd length, then $H_{\mathcal{A}_{2}}^{1, v-1}(G)$ contains $\mathbb{Z}_{2}$-torsion, and if the cycle has even length, then $H_{\mathcal{A}_{2}}^{2, v-2}(G)$ contains $\mathbb{Z}_{2}$-torsion.

Proof. " $\Rightarrow$ " If $G$ has a loop, all the cohomology groups are zero so there can be no torsion. So we can assume $G$ has no loops. If $G$ does not have a cycle of order greater than or equal to 3, then the graph obtained from $G$ by replacing multiple edges by single edges is a forest and therefore has no torsion in its cohomology [HR04]. Since deleting multiple edges does not change the cohomology groups [HR04], the cohomology of $G$ has no torsion either. 
" $\Leftarrow$ " If $G$ has no loop and contains a cycle of order greater than or equal to 3 , Lemmas 22 and 23 below imply that $H_{\mathcal{A}_{2}}^{*}(G)$ contains torsion.

4.3. The odd cycle case

LEMMA 22. If a loopless graph $G$ with $v$ vertices contains an odd cycle of length $\geq 3$, then $H^{1, v-1}(G)$ contains $\mathbb{Z}_{2}$-torsion.

Proof. Let $G$ be a loopless graph with $v$ vertices containing a cycle of length $2 s+1$ with $s \geq 1$.

It suffices to find an element $z$ in $\operatorname{ker}\left(d^{1}\right)$ of degree $v-1$ such that $2 z=0$ in $H^{1}(G)$ and $z \neq 0$ in $H^{1}(G)$. The condition $2 z=0$ in $H^{1}(G)$ is the same as $2 z \in \operatorname{Im}\left(d^{0, v-1}\right)$. Therefore our first step will be to determine $\operatorname{Im}\left(d^{0, v-1}\right)$.

$\Delta$ Matrix representation of $d^{0, v-1}$. For convenience, we label the vertices of the graph starting with the ones in the cycle. The vertices in the cycle are labeled consecutively $v_{1}$ to $v_{2 s+1}$, with the requirement that each $v_{i}$ is adjacent to $v_{i+1}$ and $v_{2 s+1}$ is adjacent to $v_{1}$. The vertices that are not in the cycle are labeled $v_{2 s+2}$ to $v_{p}$. Examples are given in Figure 3 .
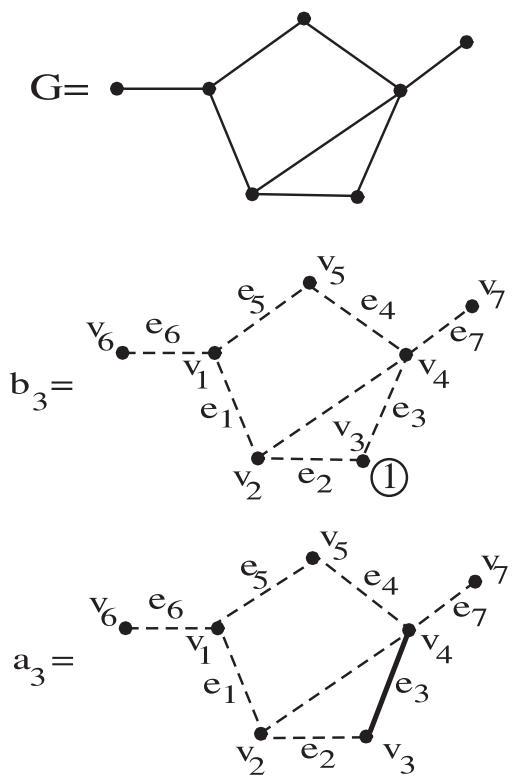

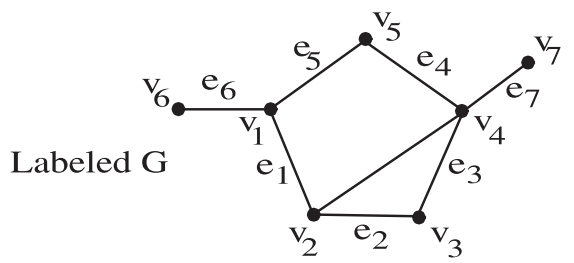

No edges

All vertices except $v_{3}$ have been assigned $x$ $\mathrm{v}_{3}$ has been assigned 1

The only present edge is $e_{3}$

All components have been assigned $\mathrm{x}$

Fig. 3. Notation for basis elements in $C^{0, v-1}(G)$ and $C^{1, v-1}(G)$

We label the edges in the cycle so that $e_{i}$ is the edge $v_{i} v_{i+1}$ for $1 \leq i \leq 2 s$ and $e_{2 s+1}$ is the edge $v_{2 s+1} v_{1}$. The edges that are not in the cycle are labeled $e_{2 s+2}$ to $e_{n}$.

We use the notion of enhanced states here. The basis elements of $C^{0, v-1}(G)$ are enhanced states $(s, c)$ where $s=\emptyset$ and $c$ is an assignment 
of 1 or $x$ to the $v$ vertices of $G$. Since the total degree is $v-1$, all vertices are assigned the color $x$ except one which is assigned the color 1 . The basis element for which 1 is assigned to the vertex $v_{i}$, and $x$ to all the other vertices, is denoted by $b_{i}$. The basis elements for which 1 is assigned to a vertex in the cycle are $b_{1}$ to $b_{2 s+1}$.

In order to write a matrix for $d^{0, v-1}$, we also need to describe the basis elements of the target space $C^{1, v-1}(G)$.

First, note that each of these basis elements contains one edge, which, by assumption, is not a loop. Therefore, each of them has $v-1$ components. Thus, for degree reasons, all the components are assigned the color $x$. The basis element for which the present edge is $e_{i}$ and with $x$ assigned to all the components is denoted by $a_{i}$. The basis elements for which the present edge is in the cycle are $a_{1}$ to $a_{2 s+1}$.

With these notations, we get a matrix representing $d^{0, v-1}$, which is shown in (2) below.

$\Delta$ Let $z=\sum_{i=1}^{n} a_{i}$.

$\triangle$ We first prove that $2 z \in \operatorname{Im}\left(d^{0, v-1}\right)$. In the matrix representation of $d^{0, v-1}$, the coordinates of the image of $b_{i}$ under the differential, in the basis $\left(a_{i}\right)_{i}$, are listed in the $i$ th row.

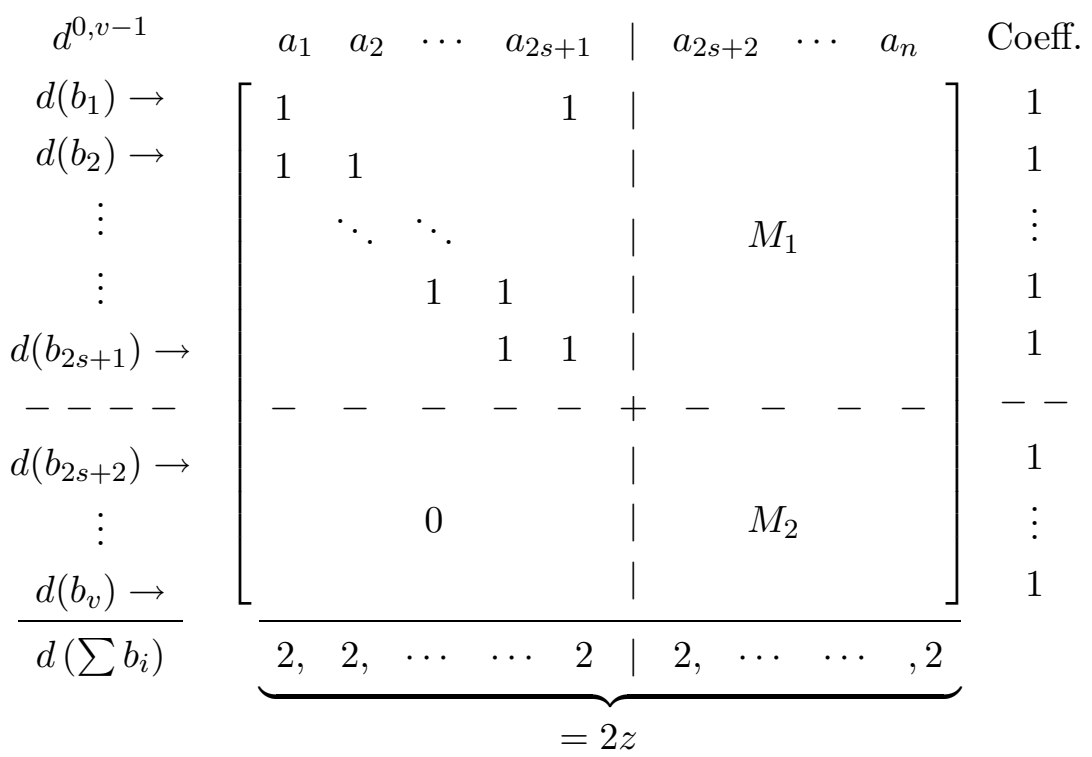

The coefficients (all equal to 1 in (2)) in the column on the right indicate the coefficient by which each line is multiplied before addition.

By adding all the rows of this matrix, we see $d\left(\sum_{i=1}^{v} b_{i}\right)=2 z$ so $2 z \in$ $\operatorname{Im}\left(d^{0, v-1}\right)$. This also implies that $z \in \operatorname{ker}\left(d^{1, v-1}\right)$. Indeed, $2 d^{1}(z)=d^{1}(2 z)=0$ in $C^{2}(G)$, which implies $d^{1}(z)=0$ since $C^{2}(G)$ has no torsion. 
The reason why adding the rows of $M_{1}$ and $M_{2}$ always yields a coordinate of 2 on the $j$ th column for $j \geq 2 s+2$ is as follows. Each edge has two ends (remember that there are no loops), so each of these $\left\{a_{j}\right\}_{j \geq 2 s+2}$ is in the image of exactly two $b_{i}$ 's, the ones corresponding to a 1 placed at each endpoint of the edge $e_{j}$.

$\triangle$ It remains to show that $z \notin \operatorname{Im}\left(d^{0, v-1}\right)$. Assume $z=d(x)$ for some $x$ in $C^{0, v-1}$. This $x$ can be written $x=\sum_{i=1}^{v} \alpha_{i} b_{i}$ for some $\alpha_{i}$.

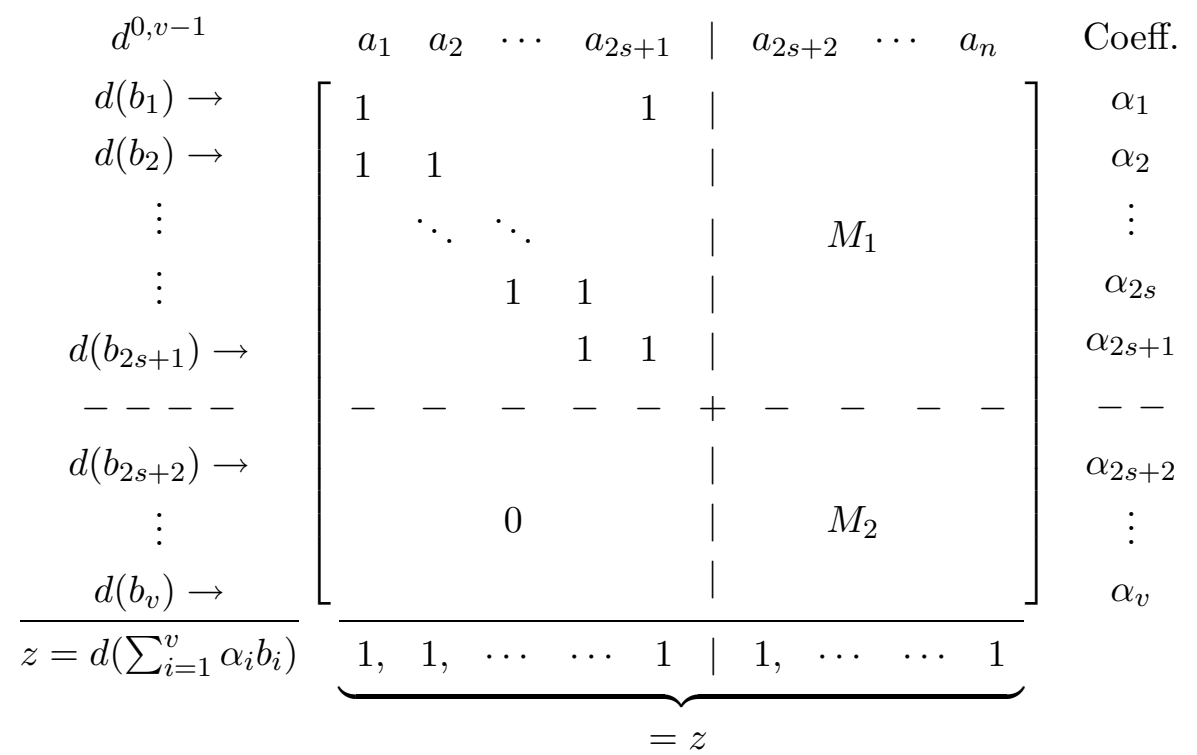

This means that the result of multiplying the first line by $\alpha_{1}$, the second line by $\alpha_{2}$, etc, and adding all the lines yields $(1,1, \ldots, 1)$, the coordinates of $z$ on the $a_{i}$ 's.

If we now read this by columns, we get a contradiction:

$$
\begin{aligned}
& \text { Col. 1: } \quad \alpha_{1}+\alpha_{2} \quad=1 \\
& \begin{array}{lll}
\text { + Col. } 2: & \alpha_{2}+\alpha_{3} & =1
\end{array} \\
& + \text { Col. } 2 s+1: \quad \alpha_{1}+\quad+\alpha_{2 s+1}=1 \\
& \underbrace{\frac{\alpha_{1}+}{2\left(\alpha_{1}+\alpha_{2}+\cdots+\alpha_{2 s}+\alpha_{2 s+1}\right)}}_{\text {even }}=\underbrace{2 s+1}_{\text {odd }}
\end{aligned}
$$

This shows that $z \notin \operatorname{Im}\left(d^{0, v-1}\right)$. 
4.4. The even cycle case. The simplest simple graph without an odd cycle is the "square" $C_{4}$. As mentioned earlier, in this case torsion appears only for $H^{2}(G)$, which indicates that we have to look deeper into the cohomology to find torsion than in the odd cycle case.

Lemma 23. Let $G$ be a simple graph, i.e. a graph with no loops and no multiple edges. If $G$ contains an even cycle of length $\geq 4$, then $H^{2, v-2}(G)$ contains $\mathbb{Z}_{2}$-torsion.

Proof. It suffices to find an element $z$ in $\operatorname{ker}\left(d^{2, v-2}\right)$ such that $2 z=0$ in $H^{2}(G)$ and $z \neq 0$ in $H^{2}(G)$. The condition $2 z=0$ in $H^{2}(G)$ means that $2 z \in \operatorname{Im}\left(d^{1, v-2}\right)$. Thus, our first step is to determine $\operatorname{Im}\left(d^{1, v-2}\right)$.

$\Delta$ Matrix representation of $d^{1, v-2}$. The labeling of the vertices and edges of the graph is the same as described in the odd cycle case, as illustrated in Figure 3 . The basis elements of $C^{1, v-2}(G)$ are enhanced states $(s, c)$ where $s$ consists of one edge and $c$ is an assignment of 1 or $x$ to the $v-1$ components of $[G: s]$. Since the total degree is $v-2$, all components are assigned the color $x$ except one that is assigned the color 1 . The basis element for which the edge is $e_{i}$ and the vertex that is assigned 1 is $v_{j}$ is denoted by $b_{i}^{j}$. An example is given in Figure 4.
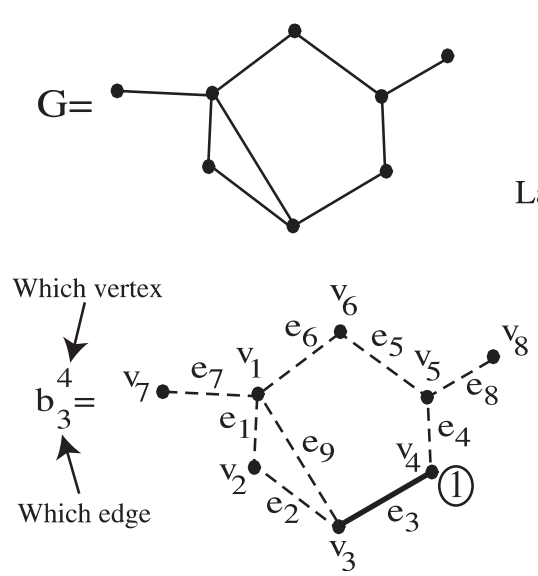

Labeled G

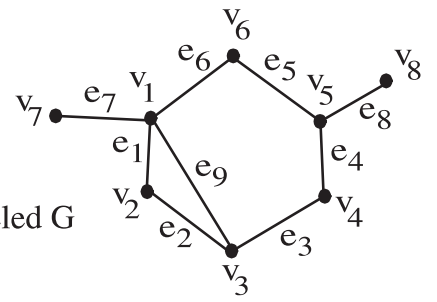

No edges except $\mathrm{e}_{3}$

All vertices except $\mathrm{v}_{4}$ have been assigned $\mathrm{x}$ $\mathrm{v}_{4}$ has been assigned 1

Fig. 4. Notation for basis elements in $C^{1, v-2}(G)$

We also need to describe the basis elements of the target space $C^{2, v-2}(G)$. Let $(s, c)$ be an enhanced state in $C^{2, v-2}(G)$. The graph $[G: s]$ has $v-2$ components, since adding two edges automatically decreases the number of components by two (note that $G$ contains no loops or multiple edges). Therefore for degree reasons, all the components are assigned the color $x$. The basis element for which the edges present are $e_{i}$ and $e_{j}$, with $x$ assigned to all components, is denoted by $a_{i j}$ with $i<j$. 
With these notations, we get a matrix representing $d^{1, v-2}$, which is shown below.

$\Delta$ We are now ready to exhibit an element $z$ in $\operatorname{ker}\left(d^{2}\right)$ of degree $v-2$ such that $2 z=0$ in $H^{2}(G)$ and $z \neq 0$ in $H^{2}(G)$.

$\triangle$ We first prove that there exists an element in $\operatorname{Im}\left(d^{1, v-2}\right)$ with all coordinates even. This is the $2 z$ we were looking for.

In the matrix representation of $d^{1, v-2}$, the coordinates of the image of $b_{i}^{j}$ under the differential, in the basis $\left(a_{i j}\right)_{i, j}$, are listed in the $i$ th row.

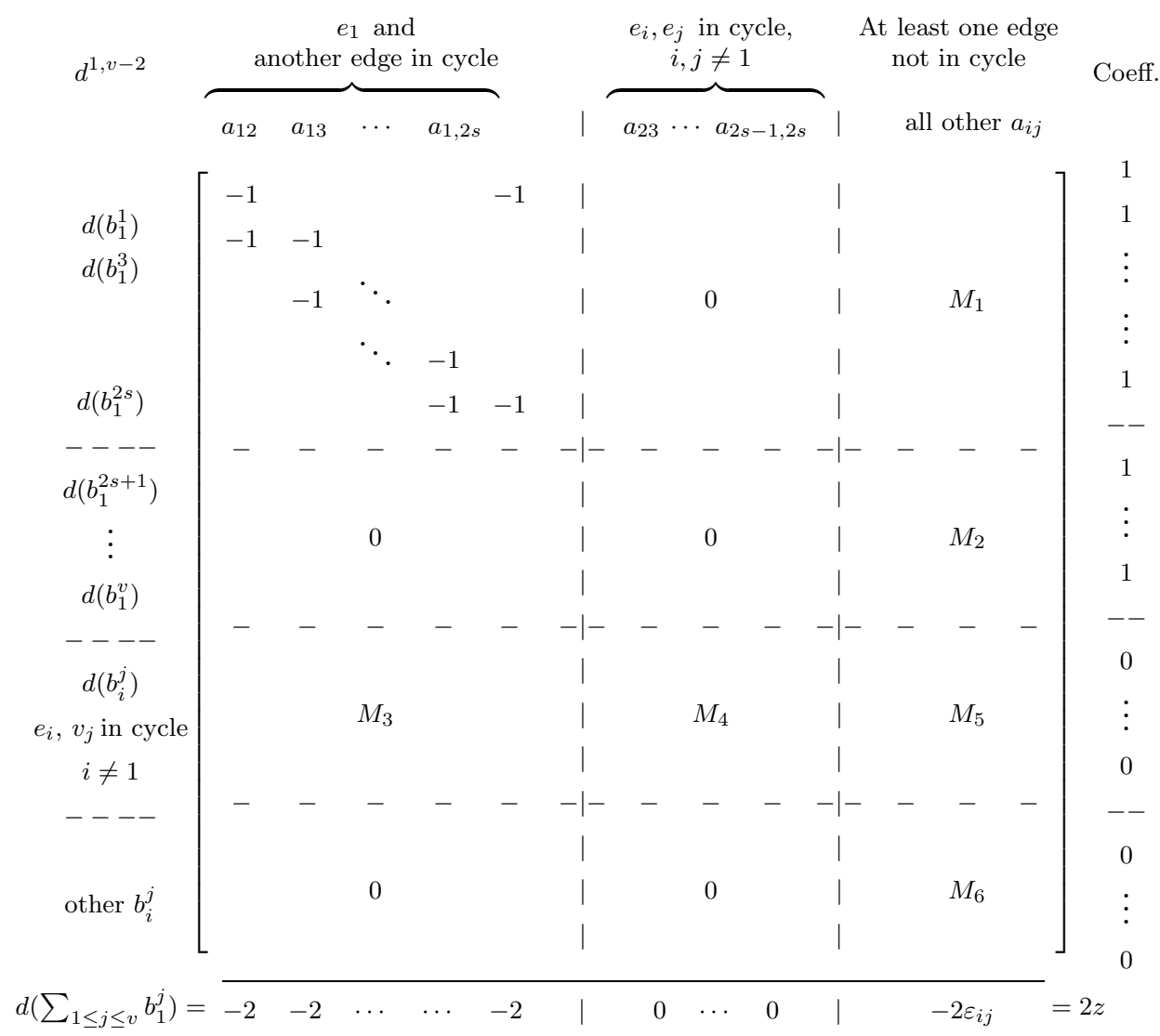

Here $\varepsilon_{i j} \in\{0,1\}$ for all $(i, j) \in J$ where $J$ is the set of all $(i, j)$ such that at least one of the edges $e_{i}, e_{j}$ is not in the cycle and $i<j$.

"Other $b_{i}^{j}$ " means either $e_{i}$ is not in the cycle or $e_{i}$ is in the cycle but $v_{j}$ is not.

We need to explain why adding the rows of $M_{1}$ and $M_{2}$ always yields a coordinate equal to -2 or 0 on each $a_{i j},(i, j) \in J$. If $i=1$, each of these $a_{1 j}$ is in the image of exactly two $b_{1}^{j}$ 's, the ones corresponding to a 1 placed 
at each endpoint of the edge $e_{j}$ (since each edge has two ends under the assumption that there are no loops). If $i \neq 1, a_{i j}$ is in the image of none of the $b_{1}^{j}$,s, hence the coordinates on the $a_{i j}$ with $i \neq 1$ are 0 .

It remains to explain the negative signs. Each $b_{1}^{j}$ is a basis element coming from the state labeled $10 \ldots 0$ (the edge present is the first one in the ordering) so the label of the per-edge map that adds the edge $i$ with $i \geq 2$ is $10 \ldots 0 * 0 \ldots 0$ with the star in the $i$ th position. The definition of the differential says that when there are an odd number of 1 's before the star, the map is assigned a negative sign.

By adding the first $v$ rows of this matrix, we see that all the coordinates of $d\left(\sum_{j=1}^{v} b_{1}^{j}\right)$ are even so we can call this element $2 z$. Hence we have achieved our first goal which was to find $2 z \in \operatorname{Im}\left(d^{1, v-2}\right)$.

Note that since $C^{3}(G)$ has no torsion, this implies that $z \in \operatorname{ker}\left(d^{2, v-2}\right)$.

$\triangle$ It remains to show that $z \notin \operatorname{Im}\left(d^{1, v-2}\right)$. Assume $z$ can be written $z=d(y)$ for some $y \in C^{1, v-2}(G)$. We write the coordinates of $y$ in the same basis of $C^{1, v-2}(G)$ used previously to write the matrix expression for $d^{1, v-2}$.

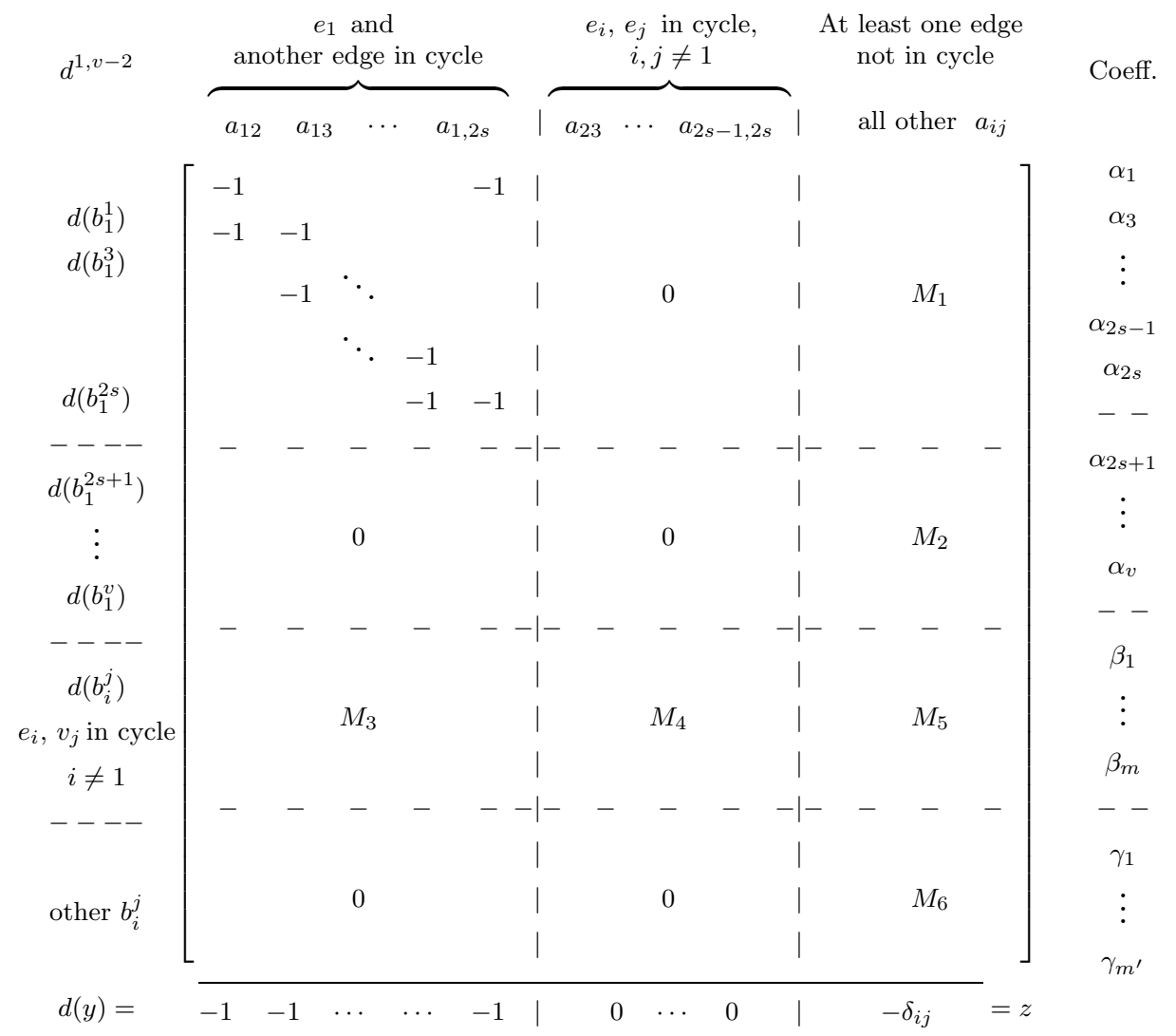


Namely, the basis can be described as a partition $B_{1} \sqcup B_{2} \sqcup B_{3}$ where $B_{1}$ is the set of basis elements for which the edge present is $e_{1}, B_{2}$ is the set of basis elements $b_{i}^{j}$ such that $e_{i}$ is an edge in the cycle but is not $e_{1}$ and $v_{j}$ is a vertex in the cycle, and $B_{3}$ is the set of all other basis elements $b_{i}^{j}$, i.e. the ones for which either $e_{i}$ is an edge in the cycle but $v_{j}$ is not a vertex in the cycle, or $e_{i}$ is not in the cycle. Using this basis, we can write $y$ as a linear combination of basis elements, labeling its coordinates on elements of $B_{1}$ by $\alpha_{i}$, its coordinates on elements of $B_{2}$ by $\beta_{i}$, and its coordinates on elements of $B_{3}$ by $\gamma_{i}$, as illustrated in the above matrix representation.

Note that there is no $b_{1}^{2}$ in $B_{1}$ since $b_{1}^{2}$ and $b_{1}^{1}$ would be the same (and no corresponding $\alpha_{2}$ coefficient). Hence the first block matrix with the -1 's is a $(2 s-1,2 s-1)$-matrix.

If we now read this by columns, what we get for the first two blocks of columns in the matrix representation, i.e. the columns corresponding to basis elements with both edges in the cycle, is the following:

$$
\begin{aligned}
& \text { Col. } 1\left(a_{12}\right): \quad-\alpha_{1}-\alpha_{3} \quad \mid \begin{array}{l}
+S_{1} \quad=-1 \\
\text { Col. } 2\left(a_{13}\right):
\end{array} \\
& \text { Col. } 2\left(a_{13}\right): \quad \begin{array}{llll}
-\alpha_{3} & -\alpha_{4} & +S_{3} & =-1
\end{array}
\end{aligned}
$$

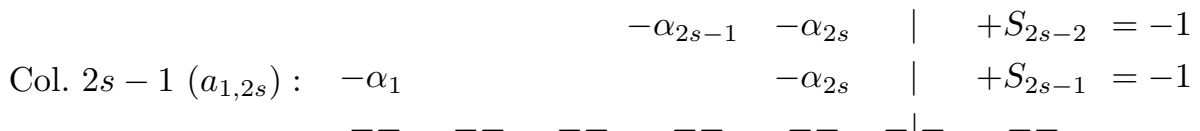

$$
\begin{aligned}
& \begin{array}{l|l}
\text { Col. } 2 s\left(a_{23}\right): & +S_{2 s}=0
\end{array} \\
& \text { Col. }\left(\begin{array}{c}
2 s \\
2
\end{array}\right)\left(a_{2 s-1,2 s}\right): \quad \begin{array}{lllll} 
& & & +S_{\left(\begin{array}{c}
2 s \\
2
\end{array}\right)}=0 \\
\hline-2\left(\begin{array}{llllll}
\alpha_{1} & +\alpha_{3} & +\alpha_{4} & +\cdots+\alpha_{2 s}
\end{array}\right) & +S_{\beta} & =2 s-1
\end{array}
\end{aligned}
$$

Here the $S_{i}$ 's are the result of the multiplication of $M_{3}$ and $M_{4}$ by the $\beta_{i}$ 's, read by columns. Their sum $S_{\beta}$ is a linear combination of $\beta_{i}$ 's with coefficients in $\mathbb{Z}$. It suffices to prove that these coefficients are all even to get a contradiction, since this will imply that the left hand side is even while the right hand side is odd. This will be achieved by showing that there are exactly two non-zero entries in each row of the matrix $M=\left[M_{3} \mid M_{4}\right]$, and that these entries are \pm 1 .

Indeed, for any $b_{i}^{j}$ with $e_{i}, v_{j}$ in the cycle, there are exactly two ways to add an edge adjacent to the component with the color 1 under the condition that this edge is in the cycle. The coordinates of $d\left(b_{i}^{j}\right)$ on these two basis elements are \pm 1 and appear in $M$. The other basis elements in the image of 
$b_{i}^{j}$ under the differential will have at least one edge not in the cycle so the corresponding coordinate will appear in $M_{5}$.

4.5. Connection with Khovanov cohomology for links. Recall that there is a bijection, originally described by Tait, between the set of unoriented alternating link diagrams and the set of plane graphs. Indeed, one can draw a crossing on each edge of a plane graph according to the diagram $\left.-1 \longrightarrow{ }^{1}\right)$, and then connect along the edges, as illustrated by the dotted lines in Figure 5. This way we obtain an unoriented alternating link diagram.

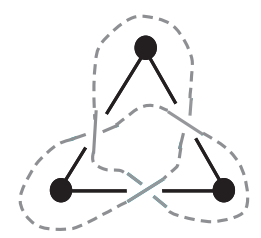

Fig. 5. The left handed trefoil knot and its Tait graph $P_{3}$

Conversely, we can obtain a plane graph from the diagram of an alternating link using the chess-board coloring according to the convention

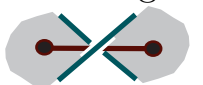

(for details see [P07]).

The version of Khovanov cohomology that we are going to use here is the one for framed unoriented links defined by Viro in Section 6 of [V04]. It categorifies the augmented Kauffman bracket polynomial, i.e. the version of the Kauffman bracket polynomial which takes the value 1 on the empty link. Since different notations have been used to describe this categorification, we first specify the ones we use. The crossings are labeled 1 to $n$ and a 0 -marker (resp. a 1-marker) associated to a crossing indicates that we perform an $A$-smoothing (resp. an $A^{-1}$-smoothing) at this crossing. An $A$-smoothing is what Khovanov calls a 0-resolution and what Bar-Natan calls a 0-smoothing. They correspond to the positive markers of Viro.

Figure 6 illustrates the relation between the chain complexes. In this figure, we adopt a presentation of our chain complex similar to the one in [BN02].

THEOREM 24. Let $D$ be the diagram of an unoriented framed alternating link and let $G$ be its Tait graph. Let $l$ be the length of the shortest cycle in $G$.

$\left({ }^{1}\right)$ Our convention of creating a link diagram from a graph corresponds to a negative graph (signed graph with all negative edges). However, we do not deal with signed graphs in this paper so our convention should not lead to confusion. Our choice is dictated by the fact that we want a 0 -resolution of the crossing in the link case to correspond to the case when the edge is absent in the graph case. 

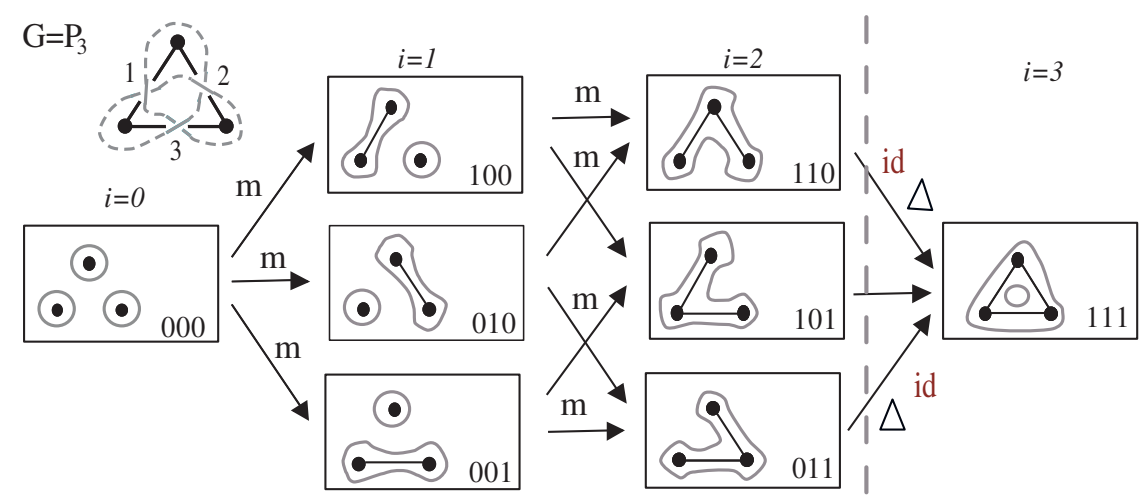

The chain complexes agree until a cycle is closed

| Then the chain complexes diverge

Fig. 6. Comparison of the chain complexes for the left handed trefoil knot and its Tait graph $P_{3}$ when the algebra is $\mathbb{Z}[x] /\left(x^{2}\right)$

For all $i<l-1$, we have

$$
H_{\text {Graph }}^{i, j}(G) \cong H_{p, q}(D) \text { with }\left\{\begin{array}{l}
p=V(G)-i-2 j, \\
q=E(G)-2 V(G)+4 j,
\end{array}\right.
$$

where $H_{p, q}$ is the homology groups of the version of Khovanov cohomology theory for unoriented framed links defined in [V04] $\left({ }^{2}\right)$.

Furthermore $\operatorname{Tor}\left(H^{i, j}(G)\right)=\operatorname{Tor}\left(H_{p, q}(D)\right)$ for $i=l-1$.

Proof. For each state $\alpha \in\{0,1\}^{n}$, as long as adding edges does not close a cycle in the graph, the connected components of the graph and link cases naturally correspond to each other. See Figure 6 for an example. Viro does not mention the $\mathbb{Z}$-algebra $\mathbb{Z}[x] /\left(x^{2}\right)$ in his construction but he assigns + and - signs to the circles and then combines them when two discs merge using the obvious multiplication: $+*+=+,+*-=-*+=-$ and $-*-=+$. With this multiplication, the free $\mathbb{Z}$-module with basis + and - is a $\mathbb{Z}$-algebra isomorphic to $\mathbb{Z}[x] /\left(x^{2}\right)$ under the correspondence $+\leftrightarrow 1,-\leftrightarrow x$. In both cases we build the chain groups by assigning a copy of $\mathcal{A}_{2}=\mathbb{Z}[x] /\left(x^{2}\right)$ to each component, taking tensor products over components and direct sum along columns. Therefore the chain groups are isomorphic as long as the spanning subgraphs are trees.

The differentials also agree: As long as adding edges does not close a cycle in the graph, changing one marker from 0 to 1 corresponds to connecting discs in the link case and to connecting components in the graph case so in

$\left(^{2}\right)$ If we use the previous definition in [V02], we get $H_{\mathrm{Graph}}^{i, j}(G) \cong H_{a, b}(D)$ with $a=E(G)-2 i, b=E(G)-2 V(G)+4 j$. In Viro's both definitions the degree is the same $q=b$ and corresponds to the $A$-degree of the Kauffman bracket polynomial. 
both cases the new labels are computed using the multiplication of $\mathcal{A}_{2}$. This is enough to see that the cohomology groups are isomorphic for $i<l-1$.

The definitions of the heights and gradings are different in these two chain complexes so we need to find a relation between them. Let $i$ be an integer with $i<l-1$. Recall that an enhanced state $S$ in the link cohomology is a smoothing of the link diagram according to markers with each of the resulting circles colored 1 or $x$. Given an enhanced state $S$ in the link cohomology, $p(S)$ is defined to be the number of 1's minus the number of $x$ 's [V04]. This can be written as $p(S)=\# 1-\# x=\# 1+\# x-2 \# x=\#$ circles $-2 j$ since in the graph cohomology the grading $j$ is the number of $x$. Also, note that since $i<l-1$, the number of circles in the link cohomology and the number of connected components of the graph in the corresponding graph cohomology are equal. Therefore, $p(S)=\# \operatorname{components}([G: s])-2 j$. Since adding edges does not close any cycle by our assumption on $i$, the number of connected components of the graph is equal to the number of vertices, $V(G)$, minus the number of edges, $i$. This proves that $p(S)=V(G)-i-2 j$.

We now deal with $q(S)$. Given an enhanced state $S$ in the link cohomology, $q(S)$ is defined to be the number of 0-resolutions of a crossing minus the number of 1-resolutions of a crossing minus $2 p(S)$ [V04]. This can be written $q(S)=\# 0$-resolutions $-\# 1$-resolutions $-2 p(S)=$ \#0-resolutions + \#1-resolutions - 2\#1-resolutions - $2 p(S)=$ \#crossings 2 (\#1-resolutions) $-2 p(S)$ since the number of crossings is equal to the number of edges of $G$ and the number of 1-resolutions is equal to $i$, the number of edges in $S$. Therefore $q(S)=E(G)-2 i-2(V(G)-i-2 j)$. This proves that $q(S)=E(G)-2 V(G)+4 j$.

Now, note that we can get a slightly better result for torsion than for the whole homology groups. Indeed, if we want to compute only the torsion part of some homology groups, we look only at the image, not the kernel of the differentials at that height and the images in chain complexes agree until $i=l-1$.

5. Computations for the algebras $\mathcal{A}_{m}$ and their deformations. One can conjecture that results similar to those of Section 4 (e.g. Theorem 21) hold generally for the algebras $\mathcal{A}_{m}$. In particular

Conjecture 25. The cohomology $H_{\mathcal{A}_{m}}^{* *}(G)$ of a graph $G$ contains a torsion part if and only if $G$ has no loops and contains a cycle of order greater than or equal to 3 . In this case, $H_{\mathcal{A}_{m}}^{* *}(G)$ has torsion of order dividing $m$.

In this section we show several examples confirming the conjecture and in particular we compute $H_{\mathcal{A}_{m}}^{i, j}\left(P_{3}\right)$.

As noted in the previous section, the graph cohomology for $\mathcal{A}_{2}$ can be used to approximate classical $s l(2)$ Khovanov cohomology. We can speculate 
that our more general calculation can be used to approximate KhovanovRozansky $\operatorname{sl}(m)$ homology [KR04] (in that case $\mathcal{A}=\mathcal{A}_{m}=\mathbb{Z}[x] /\left(x^{m}\right)$ ) or its deformation corresponding to the algebra $\mathcal{A}=\mathcal{A}_{p(x)}=\mathbb{Z}[x] /(p(x))$, where $p(x)$ is a polynomial in $x$ (compare [Gor04]). Almost no computation is completed for Khovanov-Rozansky $\operatorname{sl}(m)$ homology and speculations concern mostly its free part (compare [GuSchVa04, DGR05]). Our calculations can be very useful for predicting and verifying future results.

5.1. $H_{\mathcal{A}_{m}}^{i, j}$ for a triangle. We compute here the graph cohomology $H_{\mathcal{A}_{m}}^{i, j}\left(P_{3}\right)$, including the case of the polynomial ring $\mathcal{A}_{\infty}=\mathbb{Z}[x]$. We formulate our main theorem in two parts describing the torsion part and free part separately.

Recall that the Poincaré series of a sequence $\left\{M^{i}=\bigoplus_{j} M^{i, j}\right\}_{i}$ of graded $\mathbb{Z}$-modules, where $M^{i, j}$ is the set of homogeneous elements of $M^{i}$ of degree $j$, is defined to be the two-variable series $\sum_{i, j} t^{i} q^{j} \operatorname{dim}_{\mathbb{Q}}\left(M^{i, j} \otimes_{\mathbb{Z}} \mathbb{Q}\right)$. With our definition of the graded dimension, this can be rewritten $\sum_{i} t^{i} q \operatorname{dim}\left(M^{i}\right)$. The Poincaré series of a sequence of graded $\mathbb{Z}$-modules is a convenient way to store the free ranks of the $M^{i, j}$ 's.

Note that if two sequences $\left\{M^{i}\right\}_{i}$ and $\left\{M^{i}\right\}_{i}$ of free graded $\mathbb{Z}$-modules have the same Poincaré series, then the groups $M^{i, j}$ and $M^{i, j}$ are isomorphic for all $i, j$.

TheOREM 26. When the algebra is $\mathcal{A}_{m}$, the cohomology groups of $P_{3}$ are completely determined by:

(Torsion) $\operatorname{Tor}\left(H_{\mathcal{A}_{m}}^{*, *}\left(P_{3}\right)\right)=H_{\mathcal{A}_{m}}^{1, m}\left(P_{3}\right)=\mathbb{Z}_{m}$.

(Free) The Poincaré polynomial of $H_{\mathcal{A}_{m}^{*}}^{* *}\left(P_{3}\right)$ is equal to

$$
\left(q+q^{2}+\cdots+q^{m-1}\right)^{3}+t\left(q+q^{2}+\cdots+q^{m-1}\right) .
$$

THEOREM 27. When the algebra is $\mathcal{A}_{\infty}$, the cohomology groups of $P_{3}$ are completely determined by:

(Height 0$) H_{\mathcal{A}_{\infty}}^{0, *}\left(P_{3}\right)$ is the free abelian group $\left(\mathcal{A}^{\prime}\right)^{\otimes 3}$, where

$$
\mathcal{A}^{\prime}=\mathbb{Z}\{1\} \oplus \mathbb{Z}\{2\} \oplus \mathbb{Z}\{3\} \oplus \cdots=\bigoplus_{j=1}^{\infty} \mathbb{Z}\{j\} .
$$

(Height 1) $H_{\mathcal{A}_{\infty}}^{1}\left(P_{3}\right)=\mathcal{A}^{\prime}$, i.e. $H_{\mathcal{A}_{\infty}}^{1,0}\left(P_{3}\right)=0$ and $H_{\mathcal{A}_{\infty}}^{1, j}\left(P_{3}\right)=\mathbb{Z}$ for any $j>0$.

By Remark $6, H_{\mathcal{A}_{\infty}}^{i}\left(P_{3}\right)=0$ for all $i \geq 2$. Thus, statements (0) and (1) together are equivalent to saying that the cohomology groups $H_{\mathcal{A}_{\infty}^{* *}}^{* *}\left(P_{3}\right)$ are free abelian with Poincaré series $\left(q+q^{2}+q^{3}+\cdots\right)^{3}+t\left(q+q^{2}+q^{3}+\cdots\right)$.

It is not clear for which $\mathcal{A}$ the group $H_{\mathcal{A}}^{0, *}\left(P_{3}\right)$ is isomorphic to $\left(\mathcal{A}^{\prime}\right)^{\otimes 3}$ and $H_{\mathcal{A}}^{1, *}\left(P_{3}\right)$ is isomorphic to $\mathcal{A}^{\prime}$. 
Corollary 28. Let $P_{v}$ be a v-gon with $v \geq 3$; then

(Torsion) $\operatorname{Tor}\left(H_{\mathcal{A}_{m}}^{v-2, *}\left(P_{v}\right)\right)=H_{\mathcal{A}_{m}}^{v-2, m}\left(P_{v}\right)=\mathbb{Z}_{m}$.

(Free) The Poincaré polynomial of $H_{\mathcal{A}_{m}}^{v-2, *}\left(P_{v}\right)$ is equal to

$$
t^{v-2}\left(q+q^{2}+\cdots+q^{m-1}\right) .
$$

Proof. By Corollary $15, H_{\mathcal{A}_{m}}^{v-2}\left(P_{v}\right)=H_{\mathcal{A}_{m}}^{1}\left(P_{3}\right)$.

We prove Theorems 26 and 27 simultaneously. The idea of the proof is to analyze carefully the chain maps $0 \rightarrow C^{0, j} \stackrel{d^{0, j}}{\rightarrow} C^{1, j} \stackrel{d^{1, j}}{\rightarrow} C^{2, j} \stackrel{d^{2, j}}{\rightarrow} C^{3, j} \rightarrow 0$ for $P_{3}$ and $\mathcal{A}_{\infty}$. Recall that $d^{i, j}$ denotes the restriction of $d^{i}$ to the elements of degree $j$ of $C^{i}$. Precise knowledge of $d^{0}$ and $d^{1}$ will also allow us to obtain the result for $\mathcal{A}_{m}$.

We first describe the basis elements of $C_{\mathcal{A}_{\infty}}^{1, j}\left(P_{3}\right)=\mathbb{Z}^{3(j+1)}$. Each basis element is made up of two connected components, the one with the edge and the one consisting of the remaining vertex. Each component is colored with a power of $x$ such that the sum of the exponents is $j$ since we are dealing with degree $j$ elements. We denote by $f_{i}^{x^{a}}$ the basis element for which the edge present is $e_{i}$ and the color on the isolated vertex is $x^{a}$. The color on the other component does not need to be specified in the notation since it is forced to be $x^{j-a}$. To alleviate the notation we denote $f_{i}^{1}$ simply by $f_{i}$ for $i=1,2,3$. Examples are shown in Figure 7 .

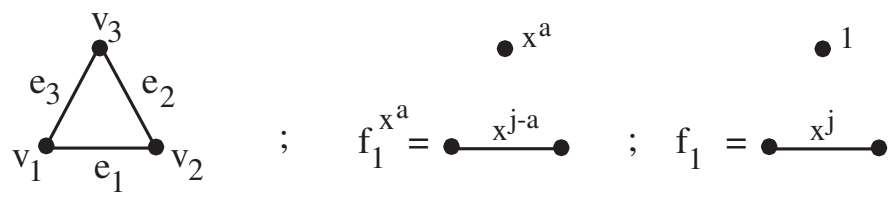

Fig. 7. Basis elements of $C_{\mathcal{A}_{\infty}}^{1, j}\left(P_{3}\right)$

Lemma 29 (Main Lemma). Consider $C_{\mathcal{A}_{\infty}}^{*, j}\left(P_{3}\right)$ with $j>0$. Then

(a) $f_{1}+f_{2}+f_{3}, f_{1}^{x^{a}}-f_{1}, f_{2}^{x^{a}}-f_{2}$ and $f_{3}^{x^{a}}-f_{3}(a \geq 1)$ form a basis of $\operatorname{ker}\left(d^{1, j}\right)$.

(b) Let $R$ denote the subgroup of $C_{\mathcal{A}_{\infty}}^{1, j}$ generated by the elements $f_{1}+$ $f_{2}+f_{3}+j\left(f_{1}^{x}-f_{1}\right)$ and all elements of the form $\left(f_{i}^{x^{a}}-f_{i}\right)-a\left(f_{1}^{x}-f_{1}\right)$ with $a \geq 1, i=1,2,3$. Then $R \subset \operatorname{Im}\left(d^{0, j}\right)$.

(c) Elements from (b) form a basis of $\operatorname{Im}\left(d^{0, j}\right)$ and in particular, $H_{\mathcal{A}_{\infty}}^{1, j}\left(P_{3}\right)=\operatorname{ker}\left(d^{1, j}\right) / \operatorname{Im}\left(d^{0, j}\right)$ is isomorphic to $\mathbb{Z}$ and generated by the class of $f_{1}^{x}-f_{1}$. Furthermore $f_{1}+f_{2}+f_{3} \equiv-j\left(f_{1}^{x}-f_{1}\right)$ in $H_{\mathcal{A}_{\infty}}^{1, j}\left(P_{3}\right)$. 
Proof. Let us first determine $C_{\mathcal{A}_{\infty}}^{0, j}\left(P_{3}\right)$ and $C_{\mathcal{A}_{\infty}}^{2, j}\left(P_{3}\right)$, and introduce notations for their basis elements.

The vertices of $P_{3}$ are labeled as in Figure 7 . The triplet $\left(x^{a}, x^{b}, x^{c}\right)$ obviously denotes the basis element of $C_{\mathcal{A}_{\infty}}^{0, j}\left(P_{3}\right)$ where the color $x^{a}$ is associated to the first vertex, $x^{b}$ is associated to the second vertex and $x^{c}$ is associated to the third vertex. It is assumed whenever we use this notation that $a+b+c=j$.

The basis elements of $C_{\mathcal{A}_{\infty}}^{2, j}\left(P_{3}\right)$ have two edges and only one connected component. Therefore, the color assigned to this component is $x^{j}$. If the two edges are $e_{i}$ and $e_{k}$, the corresponding basis element is denoted $\left(x^{j}\right)\left(e_{i}, e_{k}\right)$. It is clear that $C^{2, j}\left(P_{3}\right)=\mathbb{Z}^{3}$ with basis $\left(x^{j}\right)\left(e_{1}, e_{2}\right),\left(x^{j}\right)\left(e_{2}, e_{3}\right)$ and $\left(x^{j}\right)\left(e_{1}, e_{3}\right)$.

Proof of (a). The elements $f_{i}^{x^{a}}$ with $i=1,2,3$ and $0 \leq a \leq j$ form a basis of $C^{1, j}=\mathbb{Z}^{3(j+1)}$. We will also use the more convenient basis formed by the elements $f_{2}, f_{3}, f_{1}+f_{2}+f_{3}, f_{1}^{x^{a}}-f_{1}, f_{2}^{x^{a}}-f_{2}$ and $f_{3}^{x^{a}}-f_{3}(a>0)$. The map $d^{2, j}$ is an epimorphism with $\operatorname{ker}\left(d^{2, j}\right)=\mathbb{Z}^{2}\{j\}$ generated by $\left(q^{j}\right)\left(e_{1}, e_{2}\right)-\left(q^{j}\right)\left(e_{2}, e_{3}\right)$ and $\left(q^{j}\right)\left(e_{1}, e_{2}\right)+\left(q^{j}\right)\left(e_{1}, e_{3}\right)$. The map $d^{1, j}$ is an epimorphism onto $\operatorname{ker}\left(d^{2, j}\right)$ and in fact $d^{1, j}\left(f_{2}\right)=\left(x^{j}\right)\left(e_{1}, e_{2}\right)-\left(x^{j}\right)\left(e_{2}, e_{3}\right)$ and $d^{1, j}\left(f_{3}\right)=\left(x^{j}\right)\left(e_{1}, e_{2}\right)+\left(x^{j}\right)\left(e_{1}, e_{3}\right)$ span $\operatorname{ker}\left(d^{2, j}\right)$. Therefore the $3 j+1$ elements $f_{1}+f_{2}+f_{3}, f_{1}^{x^{a}}-f_{1}, f_{2}^{x^{a}}-f_{2}$ and $f_{3}^{x^{a}}-f_{3}(a>0)$ form a basis of $\operatorname{ker}\left(d^{1, j}\right)$.

Note that the fact that $d^{1, j}$ is surjective shows that $H_{\mathcal{A}_{\infty}}^{2, j}\left(P_{3}\right)=0$, a fact that we could also get using Theorem 4 .

Proof of (b). We need to analyze carefully the image of $d^{0, j}$. To simplify our considerations we will use $\tau$, the automorphism of $C^{i, j}$ induced by the counter-clockwise rotation by $2 \pi / 3 . d^{0, j}$ commutes with $\tau$, i.e. $\tau d^{0, j}=d^{0, j} \tau$. In fact, the action of the full dihedral symmetry group of the triangle commutes with $d^{0, j}$. We also have $\tau^{2}\left(f_{1}^{x^{a}}\right)=\tau\left(f_{2}^{x^{a}}\right)=f_{3}^{x^{a}}$. An example is shown in Figure 8.

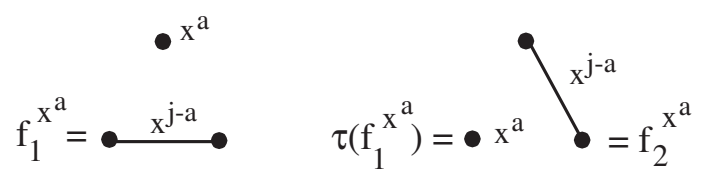

Fig. 8. Image of the basis elements of $C_{\mathcal{A}_{\infty}}^{1, j}\left(P_{3}\right)$ under $\tau$

With this in mind, consider $\operatorname{Im}\left(d_{R 1}^{0, j}\right)$ which denotes the image under $d^{0, j}$ of the subgroup $C^{0, j}(R 1)$ of $C^{0, j}$ generated by basic elements $\left(x^{a_{1}}, x^{a_{2}}, x^{a_{3}}\right)$ with at least one $a_{i}$ equal to 0 . Consider the generic element $\left(1, x^{a}, x^{b}\right)$ (all others are obtained from this one by rotation and taking different $0 \leq a \leq j$ 
with $b=j-a)$. We have

$$
d^{0, j}\left(\left(1, x^{b}, x^{a}\right)\right)=f_{1}^{x^{a}}+f_{2}+f_{3}^{x^{b}} .
$$

This relation and its image under $\tau^{2}$ with the role of $a$ and $b$ reversed, that is,

$$
d^{0}\left(\left(x^{a}, x^{b}, 1\right)\right)=f_{1}+f_{2}^{x^{a}}+f_{3}^{x^{b}}
$$

are illustrated in Figure 9.

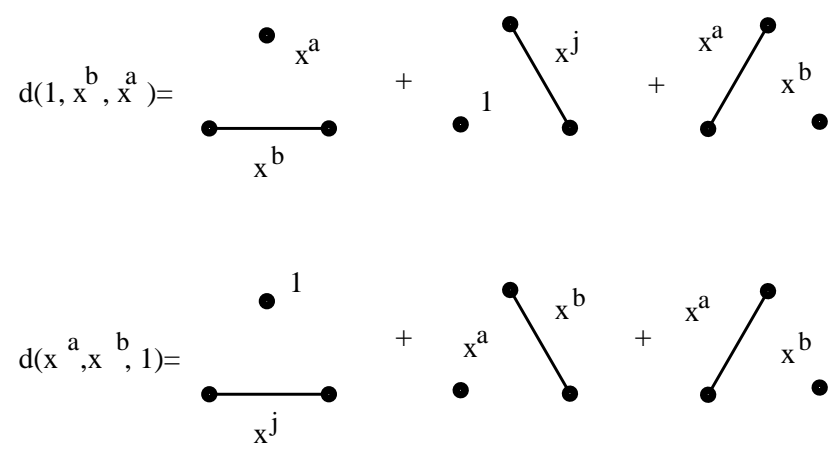

Fig. 9

Subtracting (2) from (1) shows that the elements of the form $\left(f_{1}^{x^{a}}-f_{1}\right)-$ $\left(f_{2}^{x^{a}}-f_{2}\right)$ are in $\operatorname{Im}\left(d_{R 1}^{0, j}\right)$ for any $a$. Applying $\tau$ to this relation, we find that (3) $\quad\left(f_{i}^{x^{a}}-f_{i}\right)-\left(f_{i+1}^{x^{a}}-f_{i+1}\right) \in \operatorname{Im}\left(d_{R 1}^{0, j}\right) \quad$ for any $a$ and any $i \bmod 3$.

We write

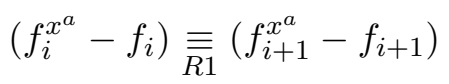

to express the fact that these terms are equal in $\operatorname{ker}\left(d^{1, j}\right) / \operatorname{Im}\left(d_{R 1}^{0, j}\right)$.

Now, note that

$$
f_{1}^{x^{a}}+f_{2}+f_{3}^{x^{b}} \underset{\overline{R 1}}{\equiv}
$$

by (1). The left hand side is equal to $f_{1}^{x^{a}}-f_{1}+f_{1}+f_{2}+f_{3}^{x^{b}}-f_{3}+f_{3}$, therefore

$$
\left(f_{1}^{x^{a}}-f_{1}\right)+\left(f_{3}^{x^{b}}-f_{3}\right)+f_{1}+f_{2}+f_{3} \underset{\overline{R 1}}{\equiv} 0 .
$$

Using (3) with $i=3$, we get

$$
\left(f_{1}^{x^{a}}-f_{1}\right)+\left(f_{1}^{x^{b}}-f_{1}\right)+f_{1}+f_{2}+f_{3} \underset{\overline{R 1}}{\equiv} 0 .
$$

From the above calculations, one can see that

$(3)^{\prime} \quad\left(f_{i}^{x^{a}}-f_{i}\right)-\left(f_{1}^{x^{a}}-f_{1}\right) \in \operatorname{Im}\left(d_{R 1}^{0, j}\right) \quad$ for any $a$ and $i=1,2,3$,

(4) $\quad\left(f_{i}^{x^{a}}-f_{i}\right)+\left(f_{i}^{x^{b}}-f_{i}\right)+f_{1}+f_{2}+f_{3} \in \operatorname{Im}\left(d_{R 1}^{0, j}\right) \quad$ for any $i$ and $a+b=j$. 
Consequently, $\operatorname{ker}\left(d^{1, j}\right) / \operatorname{Im}\left(d_{R 1}^{0, j}\right)$ is generated by the classes of $f_{1}+f_{2}+f_{3}$ and of $f_{1}^{x^{a}}-f_{1}$ for $a \leq j / 2$.

Now consider the subgroup $C^{0, j}(R 2)$ of $C^{0, j}$ generated by the enhanced states $\left(x^{a-1}, x^{b}, x\right)$ for $1 \leq a-1 \leq j-1$ with $a+b=j$. We have $d^{0, j}\left(\left(x^{a-1}, x^{b}, x\right)\right)=f_{1}^{x}+f_{2}^{x^{a-1}}+f_{3}^{x^{b}}$. Let $\operatorname{Im}\left(d_{R 1 \cup R 2}^{0, j}\right)$ denote the image under $d^{0, j}$ of the subgroup $C^{0, j}(R 1 \cup R 2)$ of $C^{0, j}$ generated by the enhanced states $\left(x^{a_{1}}, x^{a_{2}}, x^{a_{3}}\right)$ with at least one $a_{i}$ equal to 0 or to 1 . We rewrite the previous relation $f_{1}^{x}+f_{2}^{x^{a-1}}+f_{3}^{x^{b}} \equiv_{R 1 \cup R 2} 0$ to mean that the two side and the right hand sides are equal in $\operatorname{ker}\left(d^{1, j}\right) / \operatorname{Im}\left(d_{R 1 \cup R 2}^{0, j}\right)$. Adding and subtracting $f_{1}, f_{2}$ and $f_{3}$ on the left hand side leaves it unchanged so

$$
\left(f_{1}^{x}-f_{1}\right)+f_{1}+\left(f_{2}^{x^{a-1}}-f_{2}\right)+f_{2}+\left(f_{3}^{x^{b}}-f_{3}\right)+f_{3} \underset{R 1 \cup R 2}{\equiv} 0 .
$$

Applying $\left(3^{\prime}\right)$ to the terms with indices 2 and 3 yields

$$
\left(f_{1}^{x}-f_{1}\right)+\left(f_{1}^{x^{a-1}}-f_{1}\right)+\left(f_{1}^{x^{b}}-f_{1}\right)+f_{1}+f_{2}+f_{3} \underset{R 1 \cup R 2}{\equiv} 0 .
$$

We now add and subtract $f_{1}^{x^{a}}-f_{1}$ and then apply (4) since $a+b=j$. The result is $\left(f_{1}^{x}-f_{1}\right)+\left(f_{1}^{x^{a-1}}-f_{1}\right)-\left(f_{1}^{x^{a}}-f_{1}\right) \equiv_{R 1 \cup R 2} 0$, i.e.

$$
\left(f_{1}^{x^{a}}-f_{1}\right) \underset{R 1 \cup \overline{\bar{\cup}}_{R 2}}{ }\left(f_{1}^{x}-f_{1}\right)+\left(f_{1}^{x^{a-1}}-f_{1}\right) \text {. }
$$

Applying this several times we get

$$
f_{1}^{x^{a}}-f_{1} \underset{R 1 \cup R 2}{\equiv} a\left(f_{1}^{x}-f_{1}\right)
$$

and in particular

$$
f_{1}^{x^{j}}-f_{1} \underset{R 1 \cup R 2}{\equiv} j\left(f_{1}^{x}-f_{1}\right)
$$

Notice that for any $a$, applying (5) to the relation

$$
\left(f_{1}^{x^{a}}-f_{1}\right)+\left(f_{1}^{x^{b}}-f_{1}\right) \underset{R 1 \cup R 2}{\equiv}-\left(f_{1}+f_{2}+f_{3}\right)
$$

yields

$$
j\left(f_{1}^{x}-f_{1}\right) \underset{R 1 \cup R 2}{\equiv}-\left(f_{1}+f_{2}+f_{3}\right) .
$$

This completes the proof of part (b) of the lemma since it amounts to substituting (7) and $\left(3^{\prime}\right)$ in (5).

Proof of (c). We have just proved that $R \subset \operatorname{Im}\left(d_{R 1 \cup R 2}^{0, j}\right) \subset \operatorname{Im}\left(d^{0, j}\right)$, and we know from (a) that $\operatorname{ker}\left(d^{1, j}\right) / R \cong \mathbb{Z}$ is generated by the class of $f_{1}^{x}-f_{1}$.

To complete the proof of the Main Lemma we need to show that taking the quotient by $\operatorname{Im}\left(d^{0, j}\right)$ rather than $R$ does not introduce new relations, that is, $\operatorname{Im}\left(d^{0, j}\right)=R$. It is enough to observe that the images of all the basis elements $d^{0, j}\left(\left(x^{a_{1}}, x^{a_{1}}, x^{a_{1}}\right)\right)$ with $a_{1}+a_{2}+a_{3}=j$ are in $R$. In fact, in 
$\operatorname{ker}\left(d^{1, j}\right) / R$ we have

$$
\begin{aligned}
d^{0, j}\left(\left(x^{a_{1}}, x^{a_{2}}, x^{a_{3}}\right)\right) & =f_{1}^{x^{a_{3}}}+f_{2}^{x^{a_{1}}}+f_{3}^{x^{a_{2}}} \\
& \overline{\bar{R}}\left(f_{1}^{x^{a_{3}}}-f_{1}\right)+f_{1}+\left(f_{1}^{x^{a_{1}}}-f_{1}\right)+f_{2}+\left(f_{1}^{x^{a_{2}}}-f_{1}\right)+f_{3} \\
& \overline{\bar{R}} a_{3}\left(f_{1}^{x}-f_{1}\right)+a_{1}\left(f_{1}^{x}-f_{1}\right)+a_{2}\left(f_{1}^{x}-f_{1}\right)+\left(f_{1}+f_{2}+f_{3}\right) \\
& \overline{\bar{R}} j\left(f_{1}^{x}-f_{1}\right)+\left(f_{1}+f_{2}+f_{3}\right) \overline{\bar{R}} 0
\end{aligned}
$$

by $(7)$.

We are now ready to prove Theorems 26 and 27. Immediately from Lemma 29 we see that $H_{\mathcal{A}_{\infty}}^{1, j}\left(P_{3}\right)=\mathbb{Z}$ for $j>0$, which combined with $H_{\mathcal{A}_{\infty}}^{1,0}\left(P_{3}\right)=0$ shows that $H_{\mathcal{A}_{\infty}}^{1, *}\left(P_{3}\right)$ is a free abelian group with Poincaré series $t\left(q+q^{2}+\cdots\right)$.

$H_{\mathcal{A}_{\infty}}^{0, *}\left(P_{3}\right)$ can be deduced directly from $H_{\mathcal{A}_{\infty}}^{1, *}\left(P_{3}\right)$ by using the formula (1) of Section 2 that expresses the chromatic polynomial as the graded Euler characteristic of the chain complex, namely

$$
\sum_{0 \leq i \leq n}(-1)^{i} q \operatorname{dim}\left(H^{i}\right)=P_{G}(q \operatorname{dim} \mathcal{A}) .
$$

In our specific case, this becomes

$$
q \operatorname{dim}\left(H^{0}\left(P_{3}\right)\right)-q \operatorname{dim}\left(H^{1}\left(P_{3}\right)\right)=P_{P_{3}}\left(1+q+q^{2}+\cdots\right) .
$$

From this we can derive that the Poincaré series of $H_{\mathcal{A}_{\infty}}^{0, *}\left(P_{3}\right)$ is equal to $\left(q+q^{2}+\cdots\right)^{3}$.

Thus the Poincaré series of $H_{\mathcal{A}_{\infty}}^{*, *}\left(P_{3}\right)$ is $\left(q+q^{2}+\cdots\right)^{3}+t\left(q+q^{2}+\cdots\right)$. This proves Theorem 27.

In order to prove Theorem 26, we deduce the case $\mathcal{A}=\mathcal{A}_{m}$ from the case $\mathcal{A}=\mathcal{A}_{\infty}$ by adding the relation $x^{m}=0$ (see Theorem 36 for a more general setting).

For $j<m$ we have immediately $H_{\mathcal{A}_{m}}^{*, j}\left(P_{3}\right)=H_{\mathcal{A}_{\infty}}^{*, j}\left(P_{3}\right)$.

For $j=m$ we have $f_{1}=f_{2}=f_{3}=f_{1}^{x^{j}}=f_{2}^{x^{j}}=f_{3}^{x^{j}}=0$, otherwise the proof of the Main Lemma works without change. We find as before that the class of $f_{1}^{x}$ generates $\operatorname{ker}\left(d^{1}\right) / \operatorname{Im}\left(d^{0}\right)$. Furthermore from $f_{1}+f_{2}+f_{3}=0$ it follows that $j f_{1}^{x}=0$ and $H_{\mathcal{A}_{m}}^{1, j}\left(P_{3}\right)=\mathbb{Z}_{j}$.

For $j>m$ we see immediately that $\operatorname{ker}\left(d^{1, j}\right) \subset \operatorname{Im}\left(d^{0, j}\right)$. Therefore $H_{\mathcal{A}_{m}}^{1, j}\left(P_{3}\right)=0$. Theorem 26 follows.

5.2. More calculations. For a general polygon $P_{v}, v \geq 3$, one can compute $H_{\mathcal{A}_{m}}^{*, *}$ provided that we know $H_{\mathcal{A}_{m}}^{1, *}\left(P_{v^{\prime}}\right)$ for $v^{\prime} \leq \bar{v}$, as explained in Corollary 15 . The result in the classical case of $m=2$ is stated in Theorem 20 and could also be deduced from Khovanov's result on cohomology 
of $(2, v)$-torus links [K00]. We conjecture that $H_{\mathcal{A}_{m}}^{1, *}\left(P_{v}\right)$ can be described as follows $\left({ }^{3}\right)$.

CONJECTURe $30 . H_{\mathcal{A}_{m}}^{1}$ for a polygon:

$($ Odd $) \operatorname{Tor}\left(H_{\mathcal{A}_{m}}^{1, *}\left(P_{2 g+1}\right)\right)=H_{\mathcal{A}_{m}}^{1, g m}\left(P_{2 g+1}\right)=\mathbb{Z}_{m}$.

The Poincaré polynomial of $H_{\mathcal{A}_{m}}^{1, *}\left(P_{2 g+1}\right)$ is equal to

$$
t q^{(g-1) m}\left(q+q^{2}+\cdots+q^{m-1}\right) .
$$

(Even) $H_{\mathcal{A}_{m}}^{1, *}\left(P_{2 g+2}\right)$ is a free abelian group with the Poincaré polynomial equal to $t q^{g m}\left(q+q^{2}+\cdots+q^{m-1}\right)$.

We checked the conjecture in a few other specific cases in addition to $m=2$. In particular, we verified that $H_{\mathcal{A}_{3}}^{1,6}\left(P_{5}\right)=\mathbb{Z}_{3}$ and that $H_{\mathcal{A}_{3}}^{1}\left(P_{4}\right)=$ $\mathbb{Z}\{4\} \oplus \mathbb{Z}\{5\}$.

This conjecture can be used to describe in full the cohomology of $P_{v}$ via Corollary 15. Note that for $m=2$ we get Theorem 20 from Section 4 .

Conjecture 31. All cohomology groups $H_{\mathcal{A}_{m}}^{*}$ for a polygon:

(Odd) For $v=2 g+1$ we have:

$\operatorname{Tor}\left(H_{\mathcal{A}_{m}}^{*, *}\left(P_{2 g+1}\right)\right)=H_{\mathcal{A}_{m}}^{v-2, m}\left(P_{2 g+1}\right) \oplus H_{\mathcal{A}_{m}}^{v-4,2 m}\left(P_{2 g+1}\right) \oplus \cdots \oplus$ $H_{\mathcal{A}_{m}}^{1, g m}\left(P_{2 g+1}\right)$ with each summand isomorphic to $\mathbb{Z}_{m}$.

The Poincaré polynomial of $H_{\mathcal{A}_{m}}^{*, *}\left(P_{2 g+1}\right)$ is equal to

$$
\begin{aligned}
\left(q+\cdots+q^{m-1}\right)^{v}+\left(q+\cdots+q^{m-1}\right)\left(t^{v-2}\right. & +\left(t^{v-3}+t^{v-4}\right) q^{m} \\
& \left.+\left(t^{2}+t\right) q^{m(g-1)}\right) .
\end{aligned}
$$

(Even) For $v=2 g+2$ we have:

$\operatorname{Tor}\left(H_{\mathcal{A}_{m}}^{*, *}\left(P_{2 g+2}\right)\right)=H_{\mathcal{A}_{m}}^{v-2, m}\left(P_{2 g+2}\right) \oplus H_{\mathcal{A}_{m}}^{v-4,2 m}\left(P_{2 g+2}\right) \oplus \cdots \oplus$ $H_{\mathcal{A}_{m}}^{2, g m}\left(P_{2 g+2}\right)$ with each summand isomorphic to $\mathbb{Z}_{m}$.

The Poincaré polynomial of $H_{\mathcal{A}_{m}^{*}}^{*, *}\left(P_{2 g+2}\right)$ is equal to $\left(q+\cdots+q^{m-1}\right)^{v}+q^{m(v / 2)-1)}\left(q+\cdots+q^{m-1}\right)+$ $\left(q+\cdots+q^{m-1}\right)\left(t^{v-2}+\left(t^{v-3}+t^{v-4}\right) q^{m}+\left(t^{3}+t^{2}\right) q^{m(g-1)}+t q^{m g}\right)$.

The knowledge of torsion in cohomology of the triangle allows us, in some cases, to deduce existence of torsion in other graphs.

A v-gon with diagonals is a graph obtained from a polygon by adding some diagonals inside the $v$-gon in such a way that they do not intersect.

Proposition 32. If $G$ is a v-gon with diagonals and $\mathcal{A}=\mathbb{Z} 1 \oplus \mathcal{A}^{\prime}$ is an algebra satisfying Assumptions 1 , then $H_{\mathcal{A}}^{v-2, *}(G)=H_{\mathcal{A}}^{1, *}\left(P_{3}\right)$. In particular, $H_{\mathcal{A}_{m}}^{v-2, m}(G)=\mathbb{Z}_{m}$

$\left({ }^{3}\right)$ After this paper was posted on the arXiv, Conjectures 30 and 31 have been proved [P05]. 
Proof. The proposition follows by induction on the number of edges from the following two lemmas which allow the reduction of the cohomology of $G$ to the one of the triangle.

Lemma 33. Let e be an edge of $G$ with both endpoints of degree two and let $v=v_{G}>3$. Then

$$
H_{\mathcal{A}}^{v_{G / e}-2, *}(G / e)=H_{\mathcal{A}}^{v_{G}-2, *}(G) .
$$

Proof. Since $G-e$ has two pendant edges, Corollary 8(i) shows that $H_{\mathcal{A}}^{v-3, *}(G-e)=0$ and $H_{\mathcal{A}}^{v-2, *}(G-e)=0$. It follows that the long exact cohomology sequence for $G$ at the edge $e$ reduces to

$$
0=H_{\mathcal{A}}^{v-3, *}(G-e) \rightarrow H_{\mathcal{A}}^{v-3, *}(G / e) \rightarrow H_{\mathcal{A}}^{v-2, *}(G) \rightarrow H_{\mathcal{A}}^{v-2, *}(G-e)=0
$$

and the lemma follows.

Lemma 34. Let e be an edge of $G$ such that e lies on a triangle in $G$ and the vertex of the triangle opposite to e has degree two. If $v=v(G)>3$ then

$$
H_{\mathcal{A}}^{v-2, *}(G-e)=H_{\mathcal{A}}^{v-2, *}(G) .
$$

Proof. By Remark $6, H_{\mathcal{A}}^{v_{G / e^{-1, *}}}(G / e)=0$. Since $v_{G / e}=v_{G}-1$, we have $H_{\mathcal{A}}^{v_{G}-2, *}(G / e)=0$. Also, after replacing the double edge of $G / e$ by a single edge (which does not change the cohomology groups [HR05]), $G / e$ has one pendant edge, so by Corollary $8(\mathrm{i}), H_{\mathcal{A}}^{v_{G / e}-2, *}(G / e)=0$, i.e. $H_{\mathcal{A}}^{v_{G}-3, *}(G / e)$ $=0$. The long exact cohomology sequence reduces in this case to

$$
0=H_{\mathcal{A}}^{v_{G}-3, *}(G / e) \rightarrow H_{\mathcal{A}}^{v_{G}-2, *}(G) \rightarrow H_{\mathcal{A}}^{v_{G}-2, *}(G-e) \rightarrow H_{\mathcal{A}}^{v_{G}-2, *}(G / e)=0
$$

and the lemma follows.

If we relax the assumptions of Lemma 33 we get the following weaker result.

Proposition 35. Let e be an edge of $G$ with one endpoint of degree two and let $v=v_{G} \geq 3$. Then there is an epimorphism $\alpha: H_{\mathcal{A}}^{v_{G / e}-2, *}(G / e) \rightarrow$ $H_{\mathcal{A}}^{v_{G}-2, *}(G)$.

The proof of the proposition is the same as that of Lemma 33, except that we use only the right side of the exact sequence from the proof. Notice that we cannot expect $\alpha$ to be always an isomorphism; for example, for $G=P_{3}$ the group $H_{\mathcal{A}}^{0, *}(G / e)$ is free while $H_{\mathcal{A}}^{1, *}(G)$ has torsion. Proposition 35 is not even sufficient to guarantee that if $H_{\mathcal{A}}^{v_{G / e^{-2}}{ }^{*}}(G / e)$ has torsion then $H_{\mathcal{A}}^{v_{G}-2, *}(G)$ has torsion. It can, however, be used to show that for a large class of graphs (corresponding in Tait translation to non-trivial, prime algebraic link diagrams) the group $H_{\mathcal{A}}^{v_{G}-2, *}(G)$ is non-trivial and dominates the first homology group of the triangle. 
Definition 36. A $P_{n}$-series-parallel graph is a graph which can be obtained from the polygon $P_{n}$ by a finite number of the following operations: replacing an edge by two edges or subdividing an edge.

Proposition 37. If a graph $G$ is $P_{n}$-series-parallel then there is an epimorphism $H_{\mathcal{A}}^{n-2}\left(P_{n}\right) \rightarrow H_{\mathcal{A}}^{v-2}(G)$, or equivalently $H_{\mathcal{A}}^{1}\left(P_{3}\right) \rightarrow H_{\mathcal{A}}^{v-2}(G)$ since the two groups on the left are isomorphic by Corollary 15.

Proof. We note that adding a parallel edge does not change the cohomology groups [HR05], and subdividing an edge induces an epimorphism (Proposition 35). Therefore the result follows by induction on the number of edges.

Since any $P_{n}$-series-parallel graph is also a $P_{3}$-series-parallel graph, this combined with Proposition 26 implies that if a graph $G$ is $P_{n}$-series-parallel for some $n$, then $H_{\mathcal{A}_{m}}^{v-2, m}(G)$ is equal to $\mathbb{Z}_{m^{\prime}}$ or 0 , where $m^{\prime}$ is a divisor of $m$.

We can use the following proposition to show that some class of graphs, including the complete graph $K_{4}$, has torsion in $H_{\mathcal{A}_{m}}^{v-2, m}(G)$.

Proposition 38. Let e be an edge of a graph $G$. If $H_{\mathcal{A}}^{v-3, j}(G / e)$ is finite, possibly trivial, and $H_{\mathcal{A}}^{v-2, j}(G-e)$ has torsion, then $H_{\mathcal{A}}^{v-2, j}(G)$ has torsion.

Proof. This follows from the long exact sequence of graph cohomology:

$$
\rightarrow H_{\mathcal{A}}^{v-3, j}(G / e) \rightarrow H_{\mathcal{A}}^{v-2, j}(G) \rightarrow H_{\mathcal{A}}^{v-2, j}(G-e) \rightarrow H_{\mathcal{A}}^{v-2, j}(G / e)=0 .
$$

To illustrate Proposition 38, consider the complete graph $K_{4}$ and any edge $e$ of it. We know by Proposition 32 that $H_{\mathcal{A}_{m}}^{2, m}\left(K_{4}-e\right)=\mathbb{Z}_{m}$ and $H_{\mathcal{A}_{m}}^{1, m}\left(K_{4} / e\right)=\mathbb{Z}_{m}$, so we have an exact sequence $\cdots \rightarrow \mathbb{Z}_{m} \rightarrow H_{\mathcal{A}_{m}}^{2, m}\left(K_{4}\right) \rightarrow$ $\mathbb{Z}_{m} \rightarrow 0$. We conclude that $H_{\mathcal{A}_{m}}^{2, m}\left(K_{4}\right)$ is a torsion group (extension of $\mathbb{Z}_{m^{\prime}}$ by $\mathbb{Z}_{m}$, where $m^{\prime}$ is a divisor of $m$ ) and contains an element of order $m$. Recall that a group $H$ is an extension of $\mathbb{Z}_{m^{\prime}}$ by $\mathbb{Z}_{m}$ means that there is an exact sequence of the form $0 \rightarrow \mathbb{Z}_{m^{\prime}} \rightarrow H \rightarrow \mathbb{Z}_{m} \rightarrow 0$.

The graph $K_{4}$ is one of the series of graphs for which we can prove, in a similar manner, that $H_{\mathcal{A}_{m}}^{v_{G}-2, m}(G)$ has nontrivial torsion. Some of these graphs are illustrated in Figure 9.
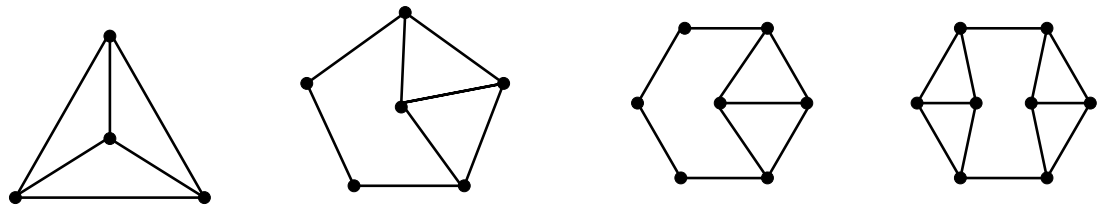

Fig. 9. Graphs with torsion in $H_{\mathcal{A}_{m}}^{v_{G}-2, m}(G)$ 
Notice that in our examples $H_{\mathcal{A}_{m}}^{v_{G}-2}(G)$ had non-trivial torsion. This is not always the case, however, at least for graphs which are not 2-connected. Consider, for example, the one-vertex product

$$
P_{3} * P_{3}=\triangleright \downarrow \text {. }
$$

Then we have $H_{\mathcal{A}}^{3}\left(P_{3} * P_{3}\right)=0$. This is a consequence of the following three-term part of the deleting-contracting long exact sequence:

$$
\begin{aligned}
0=H_{\mathcal{A}}^{2}(\triangleright) \otimes \mathcal{A}^{\prime}=H_{\mathcal{A}}^{2}(\searrow) & =H_{\mathcal{A}}^{2}(\searrow \bigcirc) \stackrel{\alpha_{*}}{\rightarrow} H_{\mathcal{A}}^{3}(\triangleright \backslash) \\
& \stackrel{\beta_{*}}{\rightarrow} H_{\mathcal{A}}^{3}(\searrow<)=H_{\mathcal{A}}^{3}(\triangleright) \otimes \mathcal{A}^{\prime} \otimes \mathcal{A}^{\prime}=0 .
\end{aligned}
$$

In a broader context one can try to generalize results obtained for $m=2$ in Section 4. One would conjecture that if a loopless graph $G$ contains a triangle then $H_{\mathcal{A}_{m}}^{1, *}(G)$ contains $\mathbb{Z}_{m}$-torsion and if a loopless graph $G$ contains a square then $H_{\mathcal{A}_{m}}^{2, *}(G)$ contains $\mathbb{Z}_{m}$-torsion.

A. Shumakovitch has conjectured that for alternating links the torsion in Khovanov homology can have only elements of order 2. We conjecture analogously that for the algebra $\mathcal{A}_{2}$ the torsion part of $H_{\mathcal{A}_{2}}^{*, *}(G)$ can have only elements of order 2 . However, as computed in [PPS06], $H_{\mathcal{A}_{3}}^{1,5}\left(K_{4}\right)=\mathbb{Z}_{3}^{2} \oplus$ $\mathbb{Z}_{6} \oplus \mathbb{Z}^{2}$ so it is not true that torsion elements of $H_{\mathcal{A}_{3}}^{* *}(G)$ have order a power of three. Furthermore, in [PPS06], the family of plane graphs $G_{1}, \ldots, G_{k}$ (see Figure 10) is analysed and there is a strong indication that for any $n$ there is a graph with torsion $\mathbb{Z}_{n}$. In particular, $H_{\mathcal{A}_{3}}^{1,13}\left(G_{1}\right)=\mathbb{Z}_{4} \oplus \mathbb{Z}_{3}^{7} \oplus \mathbb{Z}^{12}$, $H_{\mathcal{A}_{3}}^{1,21}\left(G_{2}\right)=\mathbb{Z}_{18} \oplus \mathbb{Z}_{3}^{10} \oplus \mathbb{Z}^{15}, H_{\mathcal{A}_{3}}^{1,29}\left(G_{3}\right)=\mathbb{Z}_{8} \oplus \mathbb{Z}_{3}^{15} \oplus \mathbb{Z}^{20}, H_{\mathcal{A}_{3}}^{1,37}\left(G_{4}\right)=$ $\mathbb{Z}_{10} \oplus \mathbb{Z}_{3}^{19} \oplus \mathbb{Z}^{25}, H_{\mathcal{A}_{3}}^{1,45}\left(G_{5}\right)=\mathbb{Z}_{4} \oplus \mathbb{Z}_{9} \oplus \mathbb{Z}_{3}^{22} \oplus \mathbb{Z}^{30}, H_{\mathcal{A}_{3}}^{1,53}\left(G_{6}\right)=\mathbb{Z}_{14} \oplus \mathbb{Z}_{3}^{27} \oplus \mathbb{Z}^{35}$, and $H_{\mathcal{A}_{3}}^{1,61}\left(G_{7}\right)=\mathbb{Z}_{16} \oplus \mathbb{Z}_{3}^{31} \oplus \mathbb{Z}^{40}$.
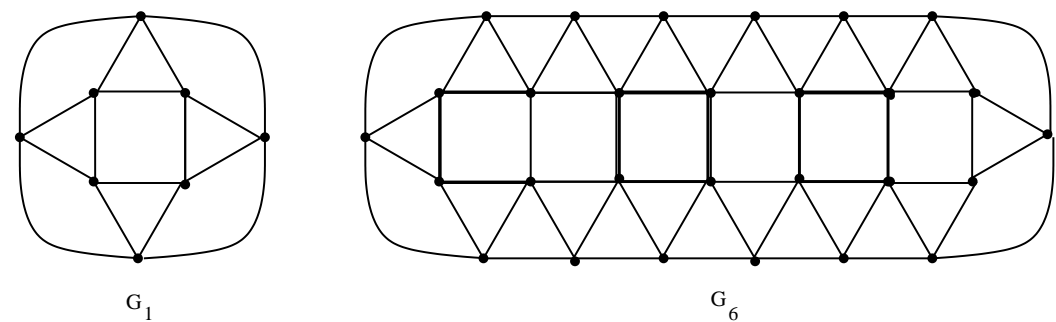

Fig. 10. Family of graphs $G_{i}$, where $i$ is the number of squares

5.3. Deformation of the algebra $\mathcal{A}_{m}$. Khovanov and Rozansky constructed $s l(m)$ homology for links [KR04]. The underlying algebra in this case was $\mathcal{A}_{m}=\mathbb{Z}[x] /\left(x^{m}\right)$. Then Gornik considered deformations of this $\operatorname{sl}(m)$ homology using as the underlying algebra $\mathcal{A}_{p(x)}=\mathbb{Z}[x] /(p(x))$, where $p$ is a polynomial. 
Here, we prove that $\left({ }^{4}\right)$ :

THEOREM 39.

(i) $H_{\mathcal{A}_{p(x)}}^{1}\left(P_{3}\right)=\mathbb{Z}[x] /\left(p(x), p^{\prime}(x)\right)$, where $p^{\prime}(x)$ is the derivative of $p(x)$ and $\left(p(x), p^{\prime}(x)\right)$ is the ideal generated by $p(x)$ and $p^{\prime}(x)$. In particular,

$$
\operatorname{rank}\left(H_{\mathcal{A}_{p(x)}}^{1}\left(P_{3}\right)\right)=\operatorname{deg}_{\mathbb{Q}} \operatorname{gcd}\left(p(x), p^{\prime}(x)\right) .
$$

Equivalently, $\operatorname{rank}\left(H_{\mathcal{A}_{p(x)}}^{1}\left(P_{3}\right)\right)=\sum_{x_{i}}\left(\operatorname{mul}\left(x_{i}\right)-1\right)$, where the sum is taken over all roots of $p(x)$ and $\operatorname{mul}\left(x_{i}\right)$ is the multiplicity of the $\operatorname{root} x_{i}$.

(ii) $H_{\mathcal{A}_{p(x)}}^{0}\left(P_{3}\right)=\mathbb{Z}^{m(m-1)(m-2)+\operatorname{deg}_{\mathbb{Q}} \operatorname{gcd}\left(p(x), p^{\prime}(x)\right)}$, where $m=\operatorname{deg} p(x)$.

(iii) More generally, consider the algebra $\mathcal{A}=\mathbb{Z}[x] / \mathbb{I}$ where $\mathbb{I}$ is an ideal in $\mathbb{Z}[x]$. Let $\mathbb{I}^{\prime}$ be the ideal generated by the derivatives of elements in $\mathbb{I}$. Note that $\mathbb{I} \subset \mathbb{I}^{\prime}$ because for any $p(x) \in \mathbb{I}$ we have $p(x)=$ $(x p(x))^{\prime}-x p^{\prime}(x)$. Then $H_{\mathbb{Z}[x] / \mathbb{I}}^{1}=\mathbb{Z}[x] / \mathbb{I}^{\prime}$.

Note that for $p(x)=x^{m}$, Theorem 39 agrees with Theorem 26 because $\mathbb{Z}[x] /\left(x^{m}, m x^{m-1}\right)$, as a group, is isomorphic to $\mathbb{Z}^{m-1} \oplus \mathbb{Z}_{m}$.

For $p(x)=x^{2}-b x-a$ we recover a result from [HR05] because

$$
\mathbb{Z}[x] /\left(x^{2}-b x-a, 2 x-b\right)=\mathbb{Z}[x] /\left(x^{2}-b x-a, 2 x-b, b x+2 a\right),
$$

which as a group is isomorphic to $\{1, x \mid 2 x-b, b x+2 a\}$ and finally to $\mathbb{Z} \oplus \mathbb{Z}_{2}$ for $b^{2}+4 a=0, \mathbb{Z}_{\left|b^{2}+4 a\right|}$ for $b^{2}+4 a \neq 0$ and $b$ odd, and $\mathbb{Z}_{2} \oplus \mathbb{Z}_{\left|b^{2}+4 a\right| / 2}$ for $b^{2}+4 a \neq 0$ and $b$ even.

For $p(x)=x^{m}-1$ we deduce from Theorem 39 that $H_{\mathcal{A}_{p(x)}}^{1}\left(P_{3}\right)=$ $\mathbb{Z}[x] /\left(x^{m}-1, m x^{m}\right)$, which is isomorphic to $\mathbb{Z}_{m}^{m}$. In fact, for $p(x)=x^{m}-1$ we conjecture more generally that $H_{\mathcal{A}_{x} m_{-1}}^{1}\left(P_{v}\right)$ is equal to 0 for even $v$ $(v \geq 2)$ and it is equal to $\mathbb{Z}_{m}^{m}$ for odd $v$.

Proof of Theorem 39. Theorem 39 follows from Lemma 29 by carefully analysing relations caused by dividing $\mathbb{Z}[x]$ by $p(x)$ or $\mathbb{I}$. To simplify the proof we introduce several useful general notions enriching the structure of $H_{\mathcal{A}}^{*}(G)$ for any $\mathcal{A}$ and $G$.

Recall that an enhanced state $S$ of $C_{\mathcal{A}}^{*}(G)$ is a spanning subgraph of $G$ with an element of $\mathcal{A}$ associated to each connected component. A typical element of $C_{\mathcal{A}}^{*}(G)$ is a linear combination of enhanced states with coefficients in $\mathbb{Z}$.

$\left.{ }^{4}\right)$ The algebra $\mathcal{A}_{p(x)}$ is not necessarily free, for example $\mathcal{A}_{2 x}=\mathbb{Z} \oplus \mathbb{Z}_{2} \oplus \mathbb{Z}_{2} \oplus \cdots$ and $\mathcal{A}_{2 x+1}$ is a torsion free but not free group. In fact, $\mathcal{A}_{2 x+1}=\mathbb{Z}[1 / 2]$. To be sure that $\mathcal{A}_{p(x)}$ is free we can assume that $p(x)$ is monic, but, as mentioned in the introduction, many basic properties of graph cohomology hold without this assumption, in fact no restrictions on $p(x)$ are needed in the proof of Theorem 39 . 
Proposition 40. For a graph $G$ and a chosen base vertex, $v_{1}$, the group $H_{\mathcal{A}}^{*}(G)$ is an $\mathcal{A}$-module as follows.

$\mathcal{A}$ acts on $C_{\mathcal{A}}^{*}(G)$ in the following way: For any $a \in \mathcal{A}$ and any enhanced state $S \in C_{\mathcal{A}}^{*}(G), a \cdot S$ is obtained from $S$ by multiplying by a the weight of the component of $[G: s]$ containing $v_{1}$. The action commutes with the boundary map $d$, therefore $H_{\mathcal{A}}^{*}(G)$ is an $\mathcal{A}$-module.

Proof. The proof is left to the reader.

We will also need the following useful tool.

Proposition 41. Let $\mathbb{I}$ be an ideal in the algebra $\mathcal{A}$. For a given graph $G$ consider the short exact sequence of chain complexes (of $\mathcal{A}$-modules)

$$
0 \rightarrow \operatorname{ker}(P) \stackrel{I}{\rightarrow} C_{\mathcal{A}}^{*}(G) \stackrel{P}{\rightarrow} C_{\mathcal{A} / \mathbb{I}}^{*}(G) \rightarrow 0
$$

where $P$ is induced by the projection $\mathcal{A} \rightarrow \mathcal{A} / \mathbb{I}$ and $I$ is the inclusion map. This sequence yields a long exact sequence of cohomology groups (and $\mathcal{A}$ modules)

$$
\cdots \rightarrow H_{\mathcal{A}, \mathbb{I}}^{i}(G) \stackrel{I_{*}}{\rightarrow} H_{\mathcal{A}}^{i}(G) \stackrel{P_{*}}{\rightarrow} H_{\mathcal{A} / \mathbb{I}}^{i}(G) \stackrel{\partial}{\rightarrow} H_{\mathcal{A}, \mathbb{I}}^{i+1}(G) \rightarrow \cdots .
$$

We call the group $\left(\mathcal{A}\right.$-module) $H_{\mathcal{A}, \mathbb{I}}^{i}(G)$ the relative graph cohomology of $G$.

We now use the exact sequence of Proposition 41 to prove Theorem 39. For a triangle $P_{3}$ and $\mathbb{I} \subset \mathbb{Z}[x]$ we have

$$
\cdots \rightarrow H_{\mathbb{Z}[x], \mathbb{I}}^{1}\left(P_{3}\right) \stackrel{I_{*}}{\rightarrow} H_{\mathbb{Z}[x]}^{1}\left(P_{3}\right) \stackrel{P_{*}}{\rightarrow} H_{\mathbb{Z}[x] / \mathbb{I}}^{1}\left(P_{3}\right) \stackrel{\partial}{\rightarrow} H_{\mathbb{Z}[x], \mathbb{I}}^{2}\left(P_{3}\right) \rightarrow \cdots
$$

In order to use this exact sequence to prove Theorem 39 we have to understand $H_{\mathbb{Z}[x], \mathbb{I}}^{2}\left(P_{3}\right)$ and $H_{\mathbb{Z}[x], \mathbb{I}}^{1}\left(P_{3}\right)$. The ring $\mathbb{Z}[x]$ is a Noetherian ring so $\mathbb{I}$ is finitely generated, say by polynomials $p_{1}(x), \ldots, p_{k}(x)$. We recall that $\left(p_{j}(x)\right)$ denotes the principal ideal generated by $p_{j}(x)$.

LEMma 42.

(i) $H_{\mathbb{Z}[x], \mathbb{I}}^{2}\left(P_{3}\right)=0$.

(ii) $H_{\mathbb{Z}[x], \mathbb{I}}^{1}\left(P_{3}\right)$ is generated by the classes of elements of the form (notation explained in Figure 11) $f_{1}^{p_{j}(x) x^{l}, 1}+f_{2}^{1, p_{j}(x) x^{l}}+f_{3}^{p_{j}(x) x^{l}, 1}$ for any $j$ and $l$, and $f_{1}^{u, w}-f_{1}^{p_{j}(x) x^{l}, 1}$ with $u w=p_{j}(x) x^{l}$ and $u$ or $w$ in $\left(p_{j}(x)\right)$, and their rotations $\tau\left(f_{1}^{u, w}-f_{1}^{p_{j}(x) x^{l}, 1}\right)=f_{2}^{w, u}-f_{2}^{1, p_{j}(x) x^{l}}$ and $\tau^{2}\left(f_{1}^{u, w}-f_{1}^{p_{j}(x) x^{l}, 1}\right)=f_{3}^{u, w}-f_{3}^{p_{j}(x) x^{l}, 1}$.

Proof. (i) In the relative cochain complex the group $C_{\mathbb{Z}[x], \mathbb{I}}^{2}$ is generated by the elements $f_{1,2}^{u}, f_{2,3}^{u}, f_{1,3}^{u}$ with $u \in \mathbb{I}$; see Figure 11. The group $\operatorname{ker}\left(d_{\mathbb{Z}[x], \mathbb{I}}^{2}\right)=\operatorname{ker}\left(C_{\mathbb{Z}[x], \mathbb{I}}^{2} \rightarrow C_{\mathbb{Z}[x], \mathbb{I}}^{3}\right)$ is generated by the elements of the form $f_{1,2}^{u}-f_{2,3}^{u}$ and $f_{1,3}^{u}+f_{2,3}^{u}, u \in \mathbb{I}$. These elements are in the image of $C_{\mathbb{Z}[x], \mathbb{I}}^{1}$; 
namely, they are equal to $d^{1}\left(f_{2}^{1, u}\right)$ and $d^{1}\left(f_{3}^{u, 1}\right)$ respectively; $u \subset \mathbb{I}$ in our considerations. Therefore $H_{\mathbb{Z}[x], \mathbb{I}}^{2}\left(P_{3}\right)=0$.

(ii) In fact, $d_{\mathbb{Z}[x], \mathbb{I}}^{1}: \operatorname{span}\left(f_{2}^{1, u}, f_{3}^{u, 1}\right) \rightarrow \operatorname{ker}\left(d_{\mathbb{Z}[x], \mathbb{I}}^{2}\right)$ is an isomorphism. The $\mathbb{Z}$-module $\operatorname{span}\left(f_{2}^{1, u}, f_{3}^{u, 1}\right)$ can be written equivalently as $\operatorname{span}\left(f_{2}^{1, p_{j}(x) x^{l}}\right.$, $\left.f_{3}^{p_{j}(x) x^{l}, 1}\right)$ for any $j$ and $l$. The cochain group $C_{\mathbb{Z}[x], \mathbb{I}}^{1}\left(P_{3}\right)$ has a natural generating set $f_{i}^{x^{l_{1}}, p_{j}(x) x^{l_{2}}}$ and $f_{i}^{p_{j}(x) x^{l_{1}}, x^{l_{2}}}$ for $i=1,2,3$ and any $j, l_{1}$ and $l_{2}$. We can decompose the group as $C_{\mathbb{Z}[x], \mathbb{I}}^{1}\left(P_{3}\right)=\operatorname{ker}\left(d_{\mathbb{Z}[x], \mathbb{I}}^{2}\right) \oplus \operatorname{span}\left(f_{2}^{1, p_{j}(x) x^{l}}\right.$, $\left.f_{3}^{p_{j}(x) x^{l}, 1}\right)$. Hence $\operatorname{ker}\left(d_{\mathbb{Z}[x], \mathbb{I}}^{2}\right)$ is generated by $f_{1}^{p_{j}(x) x^{l}, 1}+f_{2}^{1, p_{j}(x) x^{l}}+f_{3}^{p_{j}(x) x^{l}, 1}$ for any $j$ and $l$, and $f_{1}^{u, w}-f_{1}^{p_{j}(x) x^{l}, 1}$ with $u w=p_{j}(x) x^{l}$ and $u$ or $w$ in $\left(p_{j}(x)\right)$, and $f_{2}^{w, u}-f_{2}^{1, p_{j}(x) x^{l}}$ and $f_{3}^{u, w}-f_{3}^{p_{j}(x) x^{l}, 1}$. The classes of the generators of $\operatorname{ker}\left(d_{\mathbb{Z}[x], \mathbb{I}}^{2}\right)$ generate $H_{\mathbb{Z}[x], \mathbb{I}}^{1}\left(P_{3}\right)$.

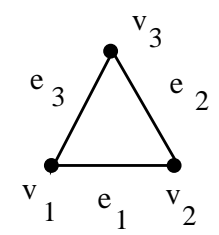

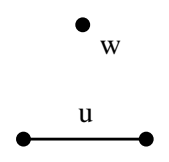

$\mathrm{f}_{1}^{\mathrm{u}, \mathrm{w}}$

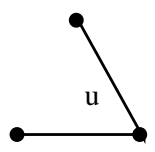

f $\underset{1,2}{u}$

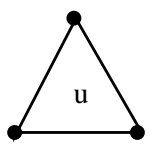

f $\underset{1,2,3}{\mathrm{u}}$

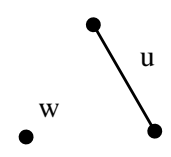

$\mathrm{f}_{2}^{\mathrm{W}, \mathrm{u}}=\tau\left(\mathrm{f}_{1}^{\mathrm{u}, \mathrm{w}}\right)$

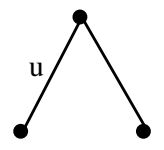

f $\underset{2,3}{\mathrm{u}}$

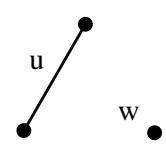

$\mathrm{f}_{3}^{\mathrm{u}, \mathrm{w}}=\tau^{2}\left(\mathrm{f}_{1}^{\mathrm{u}, \mathrm{w}}\right)$

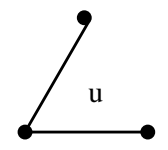

f $\underset{1,3}{\mathrm{u}}$

Fig. 11. Elements of the cochain group $C_{\mathcal{A} / \mathbb{I}}^{i}\left(P_{3}\right) ; i>0, u, v \in \mathcal{A}$. In $f_{i}^{u, v}, u$ indicates the color of the component containing $v_{1}$. 
We can now complete the proof of Theorem 39. It follows from the fact that $P_{*}$ is an epimorphism that $H_{\mathbb{Z}[x] / \mathbb{I}}^{1}\left(P_{3}\right)$ is generated by the image of the basis of $H_{\mathbb{Z}[x]}^{1}\left(P_{3}\right)$, that is,

$$
\begin{aligned}
e & =e_{1} \equiv f_{1}^{1, x}-f_{1}^{x, 1}=\stackrel{\cdot \mathrm{x}}{{ }^{1}}-\stackrel{{ }^{1}}{\longrightarrow}, \\
e_{2} & =x e \equiv f_{1}^{x, x}-f_{1}^{x^{2}, 1}, \ldots, \\
e_{j} & \equiv x^{j-1} e=f_{1}^{x^{j-1}, x}-f_{1}^{x^{j}, 1}, \ldots
\end{aligned}
$$

(notation of Figure 11).

In order to find a presentation of $H_{\mathbb{Z}[x] / \mathbb{I}}^{1}\left(P_{3}\right)$ we have to analyze relations given by $\operatorname{Im}\left(I_{*}\right)$. By Lemma $42\left(\right.$ ii) we know the generating set of $H_{\mathbb{Z}[x], \mathbb{I}}^{1}\left(P_{3}\right)$, so we will compute their images under $I_{*}$. First we check that

$$
I_{*}\left(f_{1}^{p_{j}(x), x}-f_{1}^{x p_{j}(x), 1}\right)=p_{j}(x)\left(f_{1}^{1, x}-f_{1}^{1, x}\right)=p_{j}(x) e .
$$

Then, by Lemma 29 , assuming that $p_{j}(x)=\sum_{i=0}^{m} a_{i} x^{i}$, we have

$$
\begin{aligned}
I_{*}\left(f_{1}^{1, p_{j}(x)}-f_{1}^{x p_{j}(x), 1}\right) & =f_{1}^{1, \sum a_{i} x^{i}}-f_{1}^{\sum a_{i} x^{i}, 1} \\
& =\sum_{i=0}^{m} a_{i}\left(f_{1}^{1, x^{i}}-f_{1}^{x^{i}, 1}\right) \\
& \equiv \sum_{i=0}^{m} i a_{i}\left(f_{1}^{x^{i-1}, x}-f_{1}^{x^{i}, 1}\right) \\
& =\sum_{i=0}^{m} i a_{i} e_{i}=\sum_{i=0}^{m} i a_{i} x^{i-1} e=p_{j}^{\prime}(x) e .
\end{aligned}
$$

The polynomials $p_{j}(x)$ and $p_{j}^{\prime}(x)$ (for any $j$ ) generate the ideal $\mathbb{I}^{\prime}$. Therefore $I_{*}\left(H_{\mathbb{Z}[x], \mathbb{I}}^{1}\left(P_{3}\right)\right)$ contains $I^{\prime} e$. We will now argue that $I_{*}\left(H_{\mathbb{Z}[x], \mathbb{I}}^{1}\left(P_{3}\right)\right)=\mathbb{I}^{\prime} e$; therefore $H_{\mathbb{Z}[x] / \mathbb{I}}^{1}=\mathbb{Z}[x] / \mathbb{I}^{\prime}$. Now we perform calculations checking the images of the generators of $H_{\mathbb{Z}[x], \mathbb{I}}^{1}\left(P_{3}\right)$ under $I_{*}$ :

$$
\begin{aligned}
& I_{*}\left(f_{1}^{x^{k_{1}}} p_{j}(x), x^{k_{2}}-f_{1}^{x^{k_{1}} x^{k_{2}} p_{j}(x), 1}\right)=x^{k_{1}} p_{j}(x)\left(f_{1}^{1, x^{k_{2}}}-f_{1}^{x^{k_{2}, 1}}\right) \\
& =k x^{k_{1}+k_{2}-1} p_{j}(x) e \in \mathbb{I}^{\prime} e, \\
& I_{*}\left(f_{1}^{x^{k_{1}}, x^{k_{2}} p_{j}(x)}-f_{1}^{x^{k_{1}} x^{k_{2}} p_{j}(x), 1}\right)=x^{k_{1}}\left(f_{1}^{1, x^{k_{2}} p_{j}(x)}-f_{1}^{x^{k_{2}} p_{j}(x), 1}\right) \\
& =x^{k_{1}} \sum_{i=0}^{m}\left(i+k_{2}\right) x^{i+k_{2}-1} e=k x^{k_{1}} x^{k_{2}-1} p_{j}(x) e+x^{k_{1}+k_{2}} p_{j}^{\prime}(x) e \in \mathbb{I}^{\prime} e .
\end{aligned}
$$


Similarly we deal with $\tau$ rotations of these elements. It remains to check

$$
\begin{aligned}
& I_{*}\left(f_{1}^{x^{k}} p_{j}(x), 1\right. \\
& \left.\quad=f_{2}^{1, x^{k} p_{j}(x)}+f_{3}^{x^{k}} p_{j}(x), 1\right) \\
& \quad=x^{k} p_{j}(x)\left(f_{1}^{x, 1}+f_{2}^{x, 1}+f_{3}^{x, 1}\right)-\left(f_{2}^{x^{k} p_{j}(x), 1}-f_{2}^{1, x^{k} p_{j}(x)}\right) \\
& \quad=x^{k} p_{j}(x)\left(f_{1}^{x, 1}+f_{2}^{x, 1}+f_{3}^{x, 1}\right)-k x^{k-1} p_{j}(x) e-x^{k} p_{j}^{\prime}(x) e \in \mathbb{I}^{\prime} e .
\end{aligned}
$$

The proof of Theorem 39(iii), (i) is completed. Part (ii) follows by considering the chromatic polynomial of the triangle, $\lambda(\lambda-1)(\lambda-2)$. This completes the proof of Theorem 39 .

Potentially the results and ideas used in Theorem 24 can be used to compute partially the Khovanov-Rozansky $s l(n)$ homology and its deformations as defined by Gornik. In particular, Theorem 26 potentially predicts the Khovanov-Rozansky $\operatorname{sl}(n)$ homology for the trefoil. Conjecture 31 potentially predicts the Khovanov-Rozansky $\operatorname{sl}(n)$ homology for the $(2, n)$ torus links. In particular, we can predict some torsion in Khovanov-Rozansky $s l(n)$ homology in these cases.

Our calculations also confirm the connection between graph cohomology and the Hochschild homology of the underlying algebra. Furthermore, we can also speculate on a connection between our graph cohomology of a symmetric graph and the Connes cyclic homology of algebras [P05].

\section{References}

[AP04] M. M. Asaeda and J. H. Przytycki, Khovanov homology: torsion and thickness, in: J. M. Bryden (ed.), Advances in Topological Quantum Field Theory, Kluwer, 2004, 135-166.

[BN02] D. Bar-Natan, On Khovanov's categorification of the Jones polynomial, Algebr. Geom. Topol. 2 (2002), 337-370.

[C05] M. Chmutov and S. Chmutov, e-mail communications, February 2005.

[DGR05] N. M. Dunfield, S. Gukov, and J. Rasmussen, The superpolynomial for knot homologies, arXiv:math.GT/0505662.

[Gor04] B. Gornik, Note on Khovanov link cohomology, arXiv:math.QA/0402266.

[GuSchVa04] S. Gukov, A. Schwarz, and C. Vafa, Khovanov-Rozansky homology and topological strings, arXiv:hep-th/0412243.

[H05] L. Helme-Guizon, A categorification of the chromatic polynomial, Dissertation, George Washington Univ., 2005.

[HR04] L. Helme-Guizon and Y. Rong, A categorification of the chromatic polynomial, Algebr. Geom. Topol. 5 (2005), 1365-1388.

[HR05] L. Helme-Guizon and Y. Rong, Graph cohomologies from arbitrary algebras, arXiv:math.QA/0506023.

[K00] M. Khovanov, A categorification of the Jones polynomial, Duke Math. J. 101 (2000), 359-426.

[KR04] M. Khovanov and L. Rozansky, Matrix factorizations and link homology, arXiv:math.QA/0401268. 
[PPS06] M. D. Pabiniak, J. H. Przytycki, and R. Sazdanovic, On the first group of the chromatic cohomology of graphs, in preparation.

[P05] J. H. Przytycki, When the theories meet: Khovanov homology as Hochschild homology of links, arXiv:math.GT/0509334.

[P07] - KNOTS: From combinatorics of knot diagrams to the combinatorial topology based on knots, Cambridge Univ. Press, to appear in 2007, 650 pp., arXiv:math.GT/0512630, arXiv:math.GT/0601227, arXiv:math.GT/0602264.

[S04] A. Shumakovitch, Torsion of the Khovanov homology, Geom. Topol., to appear; arXiv:math.GT/0405474.

[V02] O. Viro, Remarks on the definition of Khovanov homology, arXiv:math.GT/0202199.

[V04] - Khovanov homology, its definitions and ramifications, Fund. Math. 184 (2004), 317-342.

Department of Mathematics

The George Washington University

1922 F street NW

Washington, DC 20052, U.S.A.

E-mail: lhelmeg@yahoo.com

przytyck@gwu.edu

rong@gwu.edu

Received 12 July 2005;

in revised form 7 March 2006 